\title{
DHPV: A Distributed Algorithm for Large-Scale Graph Partitioning
}

\author{
Wilfried Yves Hamilton Adoni ( $\nabla$ adoniwilfried@gmail.com ) \\ Universite Hassan II Casablanca https://orcid.org/0000-0002-1445-8018 \\ Tarik Nahhal \\ Universite Hassan II de Casablanca Faculte des Sciences Ain Chock \\ Moez Krichen \\ Universite de Sfax \\ Abdeltif El byed \\ Universite Hassan II de Casablanca Faculte des Sciences Ain Chock \\ Ismail Assayad \\ Universite Hassan II Casablanca
}

\section{Methodology}

Keywords: Big graph, Large-scale networks, k-partition, Graph partitioning algorithms, Distributed computing, GraphX

Posted Date: August 20th, 2020

DOl: https://doi.org/10.21203/rs.3.rs-30206/v2

License: (c) (i) This work is licensed under a Creative Commons Attribution 4.0 International License. Read Full License

Version of Record: A version of this preprint was published on September 16th, 2020. See the published version at https://doi.org/10.1186/s40537-020-00357-y. 


\title{
DHPV: A Distributed Algorithm for Large-Scale Graph Partitioning
}

\author{
Wilfried Yves Hamilton Adoni • Tarik Nahhal • \\ Moez Krichen • Abdeltif El byed • Ismail Assayad
}

Received: date / Accepted: date

\begin{abstract}
Big graphs are part of the movement of "Not Only SQL" databases (also called NoSQL) focusing on the relationships between data, rather than the values themselves. The data is stored in vertices while the edges model the interactions or relationships between these data. They offer flexibility in handling data that is strongly connected to each other. The analysis of a big graph generally involves exploring all of its vertices. Thus, this operation is costly in time and resources because big graphs are generally composed of millions of vertices connected through billions of edges. Consequently, the graph algorithms are expansive compared to the size of the big graph, and are therefore ineffective for data exploration. Thus, partitioning the graph stands out as an efficient and less expensive alternative for exploring a big graph. This technique consists in partitioning the graph into a set of $\mathrm{k}$ sub-graphs in order to reduce the complexity of the queries. Nevertheless, it presents many challenges because it is an NP-complete problem. In this article, we present DPHV (Distributed Placement of Hub-Vertices) an efficient parallel and distributed heuristic for large-scale graph partitioning. An application on a real-world graphs demonstrates the feasibility and reliability of our method. The experiments carried on a 10-nodes Spark cluster proved that the proposed methodology achieves significant gain in term of time and outperforms JA-BE-JA, Greedy, DFEP.
\end{abstract}

Keywords Big graph · Large-scale networks · k-partition · Graph partitioning algorithms . Distributed computing · GraphX

W. Y. H. Adoni

LIMSAD Laboratory, Faculty of sciences, Hassan II University of Casablanca,

E-mail: adoniwilfried@gmail.com

T. Nahhal

LIMSAD Laboratory, Faculty of sciences, Hassan II University of Casablanca

M. Krichen

Faculty of CSIT, Albaha University and ReDCAD Laboratory, University of Sfax

A. El Byed

LIMSAD Laboratory, Faculty of sciences, Hassan II University of Casablanca

I. Assayad

LIMSAD Laboratory, Faculty of sciences ENSEM, Hassan II University of Casablanca 


\section{Introduction}

Graphs are ubiquitous [1] in engineering sciences because they prove to be a flexible model in the modeling of various complex phenomena emanating from various disciplines [2: biological, sociological, economic, physical and technological. A great deal of research was dedicated to improving methods of analysis for these networks 3,4. Nevertheless, the effectiveness and applicability of these methods are still limited to small networks because of the complexity of exhaustive analysis 3 . The analysis of a complex network is very expansive and consumes a lot of hardware resources because of the NP-completeness of the problem [5,6].

Large-scale network such as social networks (e.g., Facebook and Twitter) [7,8, road networks 9, 10, 11, 12, 8, brain networks [2], etc... with their heterogeneity allow to analyze a chaotic dynamics or represent a complex phenomenon. They represent numerous exciting challenges related to high performance computing problems, where data scalability, program complexity and robustness hardware configurations play an important role [13. Solving these problems can contribute to efficiently manage the new trend technologies such as big data (e.g., dataViz), distributed systems (e.g., Hadoop [14 and Spark [15) or future communication networks (e.g., $5 \mathrm{G}$ or IoT). Network analysis is widely used in various domains where experimenting relies on large-scale dataset structured as graph data and each information is stored in a vertex an the edges modelize interactions between vertices 8 .

The analysis of a large-scale network consists of exploring the properties associated with the edges and vertices of a graph. Given a large-scale network, the time complexity of the graph algorithms increases exponentially compared to the number of vertices [1]. Thus, to speed up the performance of graph algorithms, it's recommended to use distributed system to speed up analytical tasks [16. This technique is widely used in NoSQL databases. It is effective because compared to the CAP theorem [17, it ensures the consistency and availability of data. Graph-oriented databases cannot guarantee all of the properties of the CAP theorem [18]. Figure 1 shows that the partitioning of a graph ensures only the properties $\mathrm{CP}$ and $\mathrm{CA}$ of the CAP theorem:

- Consistency and Availability (CA): since the graph data is stored on a distributed system, we cannot guarantee the availability and the consistency of the dataset across the cluster at each moment.

- Consistency and Partition (CP): eventually the data stored on each partition must be consistent.

- Availability and Partition (AP): to ensure fault tolerance, the vertices and/or edges must be replicated on the nodes of the cluster.

On the other hand, for a complex network it becomes expensive to maintain the analysis requests on a single node because of the time latency and hardware requirement. To cope with this problem, the divide-and-conquer technique (divide-and-conquer) [19] can provide promising solutions. This technique consists in partitioning the graph into a set of sub-graphs and assigning them to the nodes of the cluster [18]. The first challenge of graph partitioning consists of finding a partition which minimizes the cut-edges between two subgraphs. It makes it possible to reduce the communication costs in the case of a high-performance computing [20. The second challenge is the balancing of $k$ partitions, it consists of having sub-graphs whose weights are closer. For $k=2$, it is a bi-partition of the graph. For large-scale graphs, this problem gets NP-complete [5,6]. Once we seek to break the graph into $k \geq 3$ subgraphs, in polynomial time there will be no algorithm that can resolve the problem, and there is no exact solution. If we try to partition the graph into $k>2$ subgraphs, there is no algorithm that can solve this problem in polynomial time and there is no exact solution [5,6]. 


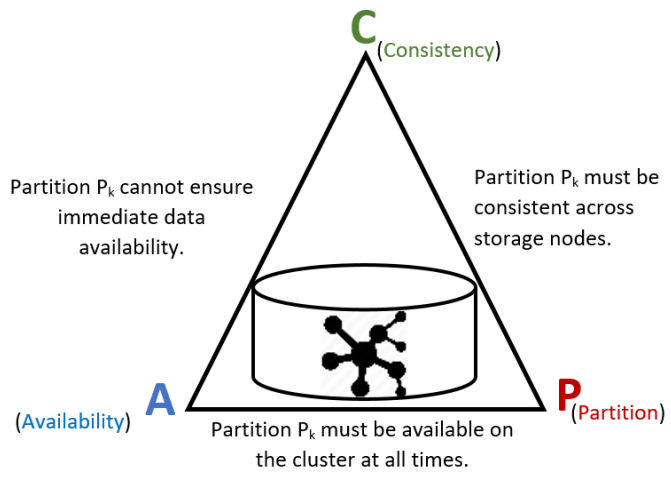

Fig. 1 CAP theorem

Partitioning means either partitioning edges or vertices [21, 8]. In general, a k-partition corresponds to vertices partitioning [8]. Figure 2 illustrates examples of a 3-partition. The colors of vertices allow to identify the classes of the partition to which they belong. A distinct color is used for each distinct class. Colored edges represent the edges connecting pairs of vertices belonging to the same subgraph and gray edges represent the cut-edges between partitions 22 . We note respectively that the partitioning in the Figure2 (a) is poor compared to that of Figure 2 (b). Note that each node stores a given partition, the cut-edges will be used for communication between nodes. Thus, a partitioning technique which minimizes the cut-edges and keeps the weight of the partitions almost equitably will thus allow to reduce the communication costs and will promote the load balancing between nodes [18.

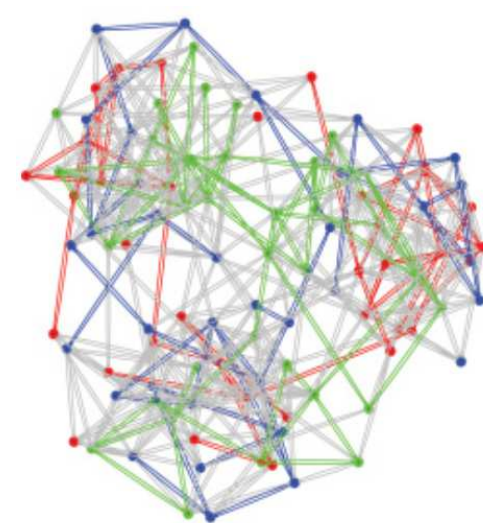

(a)

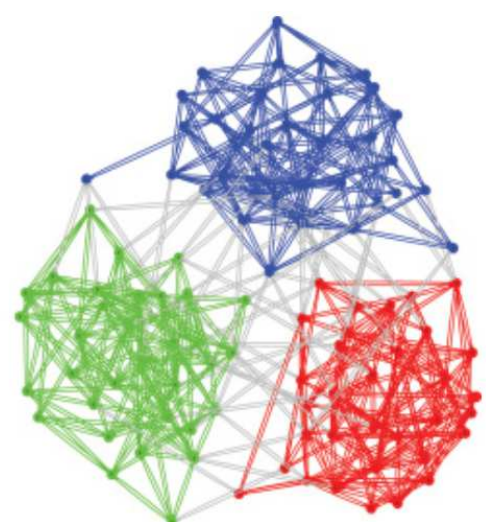

(b)

Fig. 2 Illustration of an example of partitioning : (a) the partitioning is shoddy, the vertices are assigned randomly, there are more cut-edges between partitions; (b) the partitioning is almost optimal, the highly connected vertices reside in the same partition, there is less cut-edges [22].

Contributions We present a comparative analysis of existing methods for graph partitioning. Then, we present DPHV (Distributed Placement of Hub-Vertices) a distributed algorithm for large-scale graph partitioning which meets requirements load balancing and network bandwidth of the cluster nodes [4]. The experimental results performed on a multi-nodes cluster on real-world 
graphs show that our methodology is efficient and presents conclusive results compared to current distributed graphs partitioning algorithms such as Greedy [23], DFED [21] and JA-BE-JA [24].

Organization The rest of this article is arranged as follows. We provide some required background knowledge and explain the problem formulation in Section 2. Furthermore, in Section 3, we address current methods of graph partitioning and provide a comparative analysis. Then in Section 4, we introduce our graph partitioning methodology. In Section 5, we evaluate the behavior and the performances of our method by experimental achievements. Finally, we conclude this paper with open challenges and future directions in Section 6 .

\section{Model and formalism}

In this section, we provide some basic notions related to the graph partitioning problem. Then, we present a formalism of the k-partition problem.

\subsection{Definitions and notations}

Definition 1 (Graph) A graph $G r=(V r, E d)$ is a structure made of a set of vertices $V r$ and a set of edges $E d=\left\{\left(v_{1}, v_{2}\right) \mid v_{1}, v_{2} \in V r\right\}$, which connects pairs of vertices from $V r$.

Let $|V r|$ denote the number of vertices and $|E d|$ the number of edges of the graph.

In some situations, it may be useful to assign a weight $w$ to each edge of the graph. A "weighted graph" is a graph $G r=(V r, E d, W)$ with a weighting function $W: E d \rightarrow \mathbb{R}$ associated with the set of edges.

It is worth noting that the graphs may have various topologies regarding the edge characteristics. Figure 3 proposes a comparison of different types of weighted graphs. First, we may distinguish directed and undirected graphs. A graph is said to be undirected if the edge $\left(v_{1}, v_{2}\right)$ from vertex $v_{1}$ to $v_{2}$ corresponds to the edge from $v_{2}$ to $v_{1}$. If a pair of vertices are connected by more than two edges then the graph is said to be a "multigraph". This form of graphs is more suitable for complex networks and is commonly used in NoSQL databases. An other special case of graphs is "hypergraphs", which are graphs with hyper edges connecting more than two vertices at the same time 8 .

Definition 2 (Sub-graph) Let $G r=(V r, E d)$ be a graph, $G r^{\prime}=\left(V r^{\prime}, E d^{\prime}\right)$ is a sub-graph of $G r$ if and only if $V r^{\prime}$ is a subset of $V r$ and $E d^{\prime}$ is a subset of $E d$. In other words, we obtain $G r^{\prime}$ by removing one or more vertices of $G r$, as well as all the edges incident to these vertices.

In Figure 4 (b), the graph $G r^{\prime}=\left(V r^{\prime}, E d^{\prime}\right)$ is a sub-graph of $G r=(V r, E d)$ because $V r^{\prime} \subset V r$ and $E d^{\prime} \subset E d$. We obtain $G r^{\prime}$ by removing from $G r$ the vertex 4 and these adjacent edges $(1,4)$, $(3,4)$ et $(4,5)$.

Definition 3 (Partition) Let $G r$ be a nonempty set and $k$ is a natural integer greater than or equal to 2 . We say that $P_{k}=\left\{G r_{1}, G r_{2}, \ldots, G r_{k}\right\}$ is a k-partition of $G r$ if:

- $\forall i \in \llbracket 1 ; k \rrbracket, G r_{i} \neq \emptyset$

- $\forall i, j \in \llbracket 1 ; k \rrbracket$ such as $i \neq j$, we have $G r_{i} \cap G r_{j}=\emptyset$

- $\bigcup_{i=1}^{k} G r_{i}=G r$

That is, the elements of $P_{k}$ are non-empty and pairwise disjoint. 


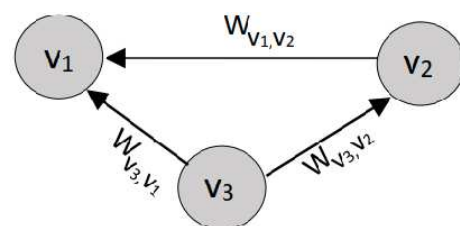

(a)

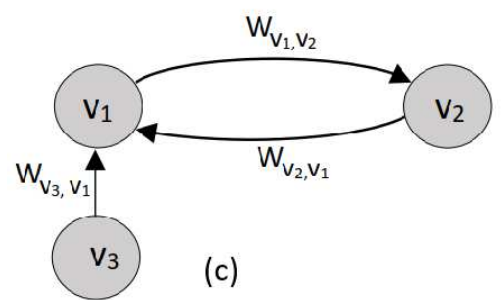

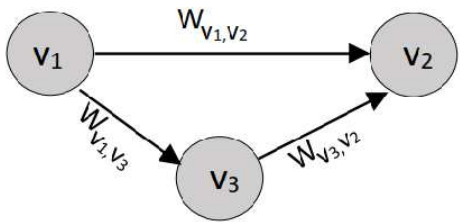

(b)

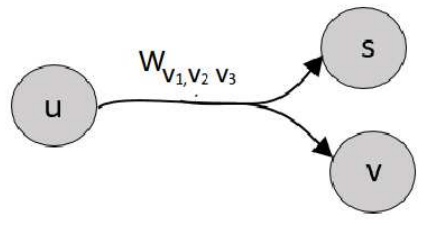

(d)

Fig. 3 Various types of graphs: (a) undirected graph; (b) directed graph; (c) multi-graph; (d) hyper-graph.

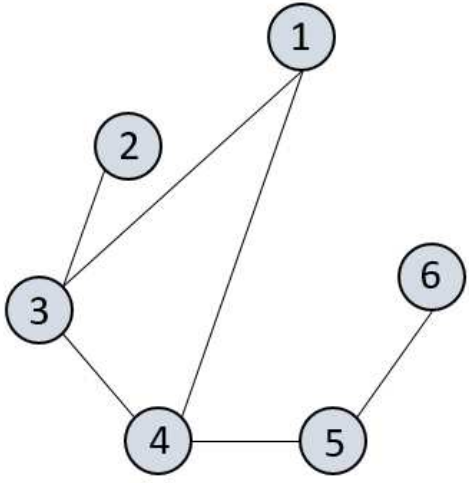

(a)

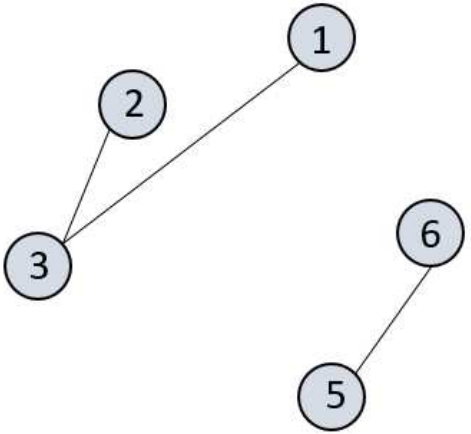

(b)

Fig. 4 (a) graph $G r=(V r, E d)$; (b) sub-graph $G r^{\prime}=\left(V r^{\prime}, E d^{\prime}\right)$.

\subsection{Formalism}

Consider a data set given in the form of a big graph $G r=(V r, E d)$ whose information is stored in $V r$ and $E d$ such that $n=|V r|$ and $m=|E d|$.

A partition $P_{k}=\left\{G r_{1}^{\prime}, G r_{2}^{\prime}, \ldots, G r_{k}^{\prime}\right\}$ of the graph $G r=(V r, E d)$ must highlight two fundamental properties:

- Balancing of sub-graphs $G r_{i}^{\prime} \forall i \in \llbracket 1 ; k \rrbracket$.

- Minimization of cuts $\operatorname{cut}\left(G r_{i}^{\prime}, G r_{j}^{\prime}\right)$ between two sub-graphs $G r_{i}^{\prime}$ and $G r_{j}^{\prime}$.

Let $w\left(G r_{i}^{\prime}\right)=\left|E d_{i}^{\prime}\right|$ be the weight of the $i^{\text {th }}$ sub-graph of $G r$, of average weight $w_{\text {avg }}$ such that:

$$
w_{\text {avg }}=\frac{\sum_{i=1}^{k} w\left(G r_{i}^{\prime}\right)}{k}
$$

The load balancing $B\left(P_{k}\right)$ of $k$ partitions consists of calculating a k-partition $P_{k}=\left\{G r_{1}^{\prime}, G r_{2}^{\prime}, \ldots, G r_{k}^{\prime}\right\}$ of $G r$ such as the weight of each sub-graph $G r_{i}^{\prime}, \forall i \in \llbracket 1 ; k \rrbracket$ contains at most $(1+\epsilon) \cdot \frac{n}{k}$ vertices. 
A partition $P_{k}$ is balanced if the constraint $B\left(P_{k}\right) \leq(1+\epsilon)$ holds, that means the size of the sub-graphs are proportionally uniform with respect to a deviation error $\epsilon$.

A better way to measure if the weights of the sub-graphs are uniformly balanced is to use the standard deviation. It is a metric that measures the dispersion of the weights of the sub-graphs. It is defined as the quadratic mean of the deviations from the mean partition. It is calculated as follows :

$$
B\left(P_{k}\right)=\sqrt{\frac{\sum_{i=1}^{k}\left(\frac{w\left(G r_{i}^{\prime}\right)}{|E d| / k}-1\right)^{2}}{k}}
$$

Lemma (Dispersion of partition weights) Given a $k$-partition of a graph, the constraint of load balancing $B\left(P_{k}\right)$ is not respected if for all sub-graphs $G r_{1}^{\prime}, \ldots, G r_{k}^{\prime}, \ldots G r_{k}^{\prime}$ of respective weight $w\left(G r_{1}^{\prime}\right), \ldots, w\left(G r_{k}^{\prime}\right), \ldots w\left(G r_{k}^{\prime}\right)$, there is a sub-graph $G r_{i}^{\prime}$ whose weight causes the imbalance of $B\left(P_{k}\right)$ such that $\forall i, j \in \llbracket 1 ; k \rrbracket$, we have:

$$
w\left(G r_{i}^{\prime}\right)>|E d|\left(\frac{\sqrt{k}}{k}(1+\epsilon)+1\right)-\sum_{j \neq i}^{k} w\left(G r_{j}^{\prime}\right)
$$

Proof In the case of a dispersion of the partition weights we have $B\left(P_{k}\right)>1+\epsilon$

The second constraint is that of cut-edges, it consists of computing a partition $P_{k}$ which allows to minimize the cut-edges between two partitions $G r_{i}^{\prime}$ and $G r_{j}^{\prime}$. Moreover, it allows to reduce the communication costs in the case of a high-performance computation. It is calculated as follows:

$$
\operatorname{cut}\left(G r_{i}^{\prime}, G r_{j}^{\prime}\right)=\sum_{s_{i} \in G r_{i}^{\prime}, s_{j} \in G r_{j}^{\prime}}\left(s_{i}, s_{j}\right)
$$

where $\left(s_{i}, s_{j}\right)$ corresponds to the cut between the sub-graphs $G r_{i}^{\prime}$ and $G r_{j}^{\prime}$. In this case, the overall cost of cut-edges the $k$ sub-graphs is calculated as follows:

$$
\operatorname{cut}\left(P_{k}\right)=\sum_{i, j \leq k} \operatorname{cut}\left(G r_{i}^{\prime}, G r_{j}^{\prime}\right)
$$

The mathematical model of the k-partition problem with constraints could be encoded using the following system [8]:

$$
\begin{cases}\min & \operatorname{cut}\left(P_{k}\right) \\ \text { subjects to: } & B\left(P_{k}\right) \leq(1+\epsilon) \\ & G r_{i}^{\prime} \cap G r_{j}^{\prime}=\emptyset \\ & \bigcup_{i=1}^{k} G r_{i}^{\prime}=P_{k} \\ & G r_{i}^{\prime}, G r_{j}^{\prime} \neq \emptyset \\ & i \neq j \in \llbracket 1 ; k \rrbracket\end{cases}
$$




\section{Related works}

For some time now, the graph partitioning problem has aroused more interest because of NP-completeness 5 of the problem. Thus, numerous algorithms appeared 3. In a survey paper, Adoni et al. 8 presented two search techniques: "local" or "global". Local search algorithms begin with an arbitrarily chosen preliminary partition to progress towards a global graph partitioning ("vertex-centric" and "edge-centric") 21]. The downside of this strategy is that the initial choice influences the quality of the obtained results [8]. In comparison, the global search approaches are based on the entire graph ("partition-centric" [21]).

The performance of graph partitioning algorithms is based on the time complexity or the result quality 5. There are extremely fast algorithms whose solution is not optimal and slow algorithms which provide solutions close to the optimal. Adoni et al. 8] classified graph analysis algorithms into different categories as shown in Figure 5.

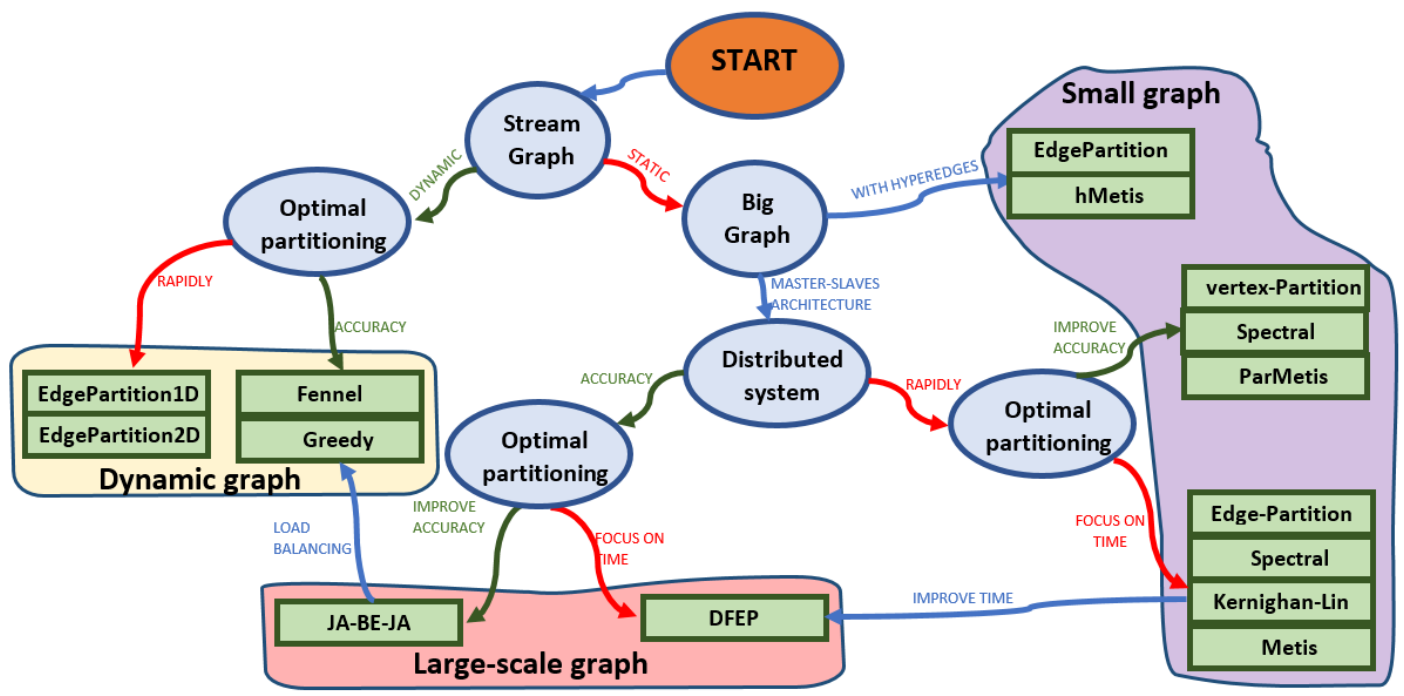

Fig. 5 Roadmap of graph partitioning algorithms

The first category concerns classical methods, there are three mains: "vertex-partition", "edge-partition" and "hypergraph-partition" [8,21]. Vertex-partition consists of partitioning the set of vertices $\operatorname{Vr}$ [5]. The cut-edges between subgraphs are used as communication channels between cluster nodes. On the other hand, edge-partition partitions the set $E d$ of edges of the graph. So, the frontier vertices are used for information exchange across the cluster 21. Vertex-partition method allows to have balanced partition $B\left(P_{k}\right)$ while edge-partition minimizes the cut-edges. An extended version of edge-centric called hypergraph-partition concerns the partitioning of hypergraphs [21]. This algorithm works as edge-partition but used the hyper-edges as cut-edges [8].

The second one concerns the spectral clustering methods [25,26. Given a set of points $\left\{x_{1}, x_{2}, \ldots, x_{n}\right\} \in \mathbb{R}^{n}$, we consider an "affinity graph" $G r=(V r, E d)$ such as the vertex $s_{i} \in V r$ corresponds to the points $x_{i} \in \mathbb{R}^{n}$. The set of edges denotes the affinities between points and the weights related to each edge $\left(s_{i}, s_{j}\right) \in E d$ encodes similarity values between $x_{i}$ and $x_{j}$. Spectral algorithm consists of steps. In the first step, we compute an affinity matrix $A$. Then, we compute 
the Laplacian matrix from $A$. Afterward, we extract the eigenvectors of $L$. Finally, we use these vectors for structural clustering.

Other algorithms [21,27,28, are based on partition via exchange. These algorithms are based on the optimization function. The choice of the initial solution influences the optimality of the result. Consequently, we may possibly fall into a local minima. Kernighan [27] used this concept to exchange the set of vertices of a given graph between k-partition while minimizing the cut-edges. Then we repeat the same task until, there are no exchanges that optimize the cut-edges function. Similarly, Fiduccia [28] presented an adapted version of "Kernighan algorithm" [27] for hypergraphs partitioning. Compared to Kernighan algorithm, it optimizes the hyper-edges function of the k-partition.

In another related works, the authors introduced [29,30,31,32,27] multilevel partitioning methodology which may be adopted for partitioning the graph into subgraphs at each level. Karypis [30 follows this concept and proposed Metis. It is made of three steps: "coarsening" step, "partitioning" step and "refinement" step. Similarly, the authors proposed hMetis 31, an extended version of Metis 30] for multilevel partition of hypergraphs. Then, they introduced a parallel version of Metis that runs on multi-core processor 32 .

Other graph partitioning algorithms are based on heuristic methods [8, 33, 15]. They are fast solving approaches but the result quality is not guaranteed to be optimal. As representative examples, we present EdgePartition1D and EdgePartition2D implemented in GraphX [15, 33. These algorithms are fast and improved version of edge-partition which optimizes cut-edges using a hash function for edges partitioning. The partitioning strategy of this heuristic is the de-randomization of edge-partition which minimizes the cut-edges between subgraphs [8].

In addition to graph algorithms, some authors [34, 35, 23] introduced streaming algorithms [36] designed for partitioning dynamic graphs. Generally, dynamic graphs 36 are subject to frequent CRUD operations over the set of vertices and edges. Unfortunately, only a few number of methods [8, 34, 35, 23, are dedicated to dynamic graphs. Aggarwal et al. 35] proposed a clustering method for graph streams. They introduced a hash function based on the compression of new edges to improve the graph clustering. In the same way, Charalampos et al 34 proposed "Fennel", a streaming graph partitioning algorithm. Fennel is founded on the optimization of an objective function which balances the weights of the subgraphs. Likewise, Joseph et al [23] presented a streaming algorithm based on power-law degree of distribution [8, 36. The proposed strategy consists of giving priority to hub-vertices.

In the end, the last category concerns distributed partitioning algorithms [22, 24, 37, 21, 38. Distributed algorithms are more effective for large-scale graphs because partitioning tasks are spread over cluster nodes. JA-BE-JA [22,24] is a successful example of distributed algorithm and its implements a local search method that is based on simulated annealing [39. It is fully decentralized, this allows the algorithm to be easily implemented into a distributed master-slaves architecture. The experimental results showed that JA-BE-JA is fast and the partition is balanced with less cut-edges. In spite of the performance of JA-BE-JA, it requires several hundred of iterations to converge towards an optimal partitioning. Therefore, it evolves costly communication overhead across the cluster nodes. To deal with this issue, Alessio [21] introduced DFEP, a distributed funding-edge partitioning algorithm. DFEP strategy consists of funding each subgraph by buying the edges of the graph at each iteration. DFEP requires less iterations to converge as compared to JA-BE-JA [24]. 


\section{Methodology}

As presented in the previous section, there are several graph partitioning techniques. Some algorithms are fast but ineffective for use cases where the result optimality is more important than the time complexity. Likewise, there are very slow algorithms which provide almost optimal results. Until then, the partitioning techniques not yet studied are the parallel and distributed approaches [40. For the moment, JA-BE-JA [22,24 is the best big graph partitioning technique but its approach based on simulated annealing is much more iterative 39. In some cases, it will be necessary to wait several hundred iterations in order to obtain a result within the limits of satisfaction constraints. Which can be very costly on time and consumes a lot of hardware resources.

In this section, we introduce DPHV (Distributed Placement of Hub-Vertices), a distributed and parallel heuristic suited for partitioning of large-scale graph according to vertex-centric paradigm and uses a monitoring agent which ensures that the weight constraints of each partitions is within normal limits. DPHV is scalable, designated for intensive computation. The partitions are strongly connected inside. In addition, it can also be implemented according to the partition-centric paradigm [40].

The proposed algorithm is parallel and distributed in a multi-nodes cluster. DPHV is based on the placement of hub-vertices. The objective of this approach is to propose partitioning according to the following criteria [3]:

- Partition balancing [41]: the partition weights must be as close as possible. This makes it possible to have the same computational loads on each node.

- Communication costs [42,38]: the exchange of information between two partitions is done through cut-edges. Each partition cut increases communication costs, which risks causing a network bottleneck in the event of a high number of cuts. Our goal is to minimize these cuts.

- Connectivity [37]: the sub-graphs induced in each partition must remain connected as much as possible as well as the clicks. This condition is not a necessity but it allows to preserve the topology of the original graph.

The balancing of the weights of the partitions can be done simply by a random placement of the vertices so as to have partitions of weight close to $\frac{|E d|}{k}[8$. However, this will involve serious communication costs between partitions and not guarantee that the topology of the graph will be preserved [13. The proposed approach takes these two compromises into account. Since the partitioning problem is considered as an NP-complete problem because of the fact that there is no exact resolution method in polynomial time. The applicability of this problem in the case of a large-scale graph is expensive and the computation time is considered impractical [5]. Generally, it takes several iterations to converge towards a quasi-optimal solution 21. It is also important to emphasize that the choice of the initial solution can lead to a local optimal problem. For example, partitions that start near the center of the graph will tend to explore more space than partitions that start at the edges of the graph.

To face these challenges, we introduced DPHV, an algorithm based on the placement of hub vertices, that is to say the vertices which have a great impact on the weight and the topology of the graph [8]. DPHV is based on vertex-partition method and implemented according to vertex-centric paradigm [24. It is an iterative algorithm which at each iteration places $k$ vertices on $k$ partitions. DPHV is completely decentralized, each slave node is responsible for placing the vertex which will cause fewer cut-edges, while the master node is responsible for coordinating and monitoring the partitioning so as to have partitions of almost similar weight [13. The vertices are sorted according to the order of their degree in the pre-processing phase, this allows derandomization of the placement and avoids local optimum problems. The hub-vertices that 
is to say having a high degree are placed as a priority. This also allows to change the graph exploration strategy. Unlike other partitioning algorithms that explore from boundaries to the center of the graph, DPHV explores in the direction of the hub vertices towards the vertices less impacting on the topology of the original graph [13. This allows in some cases to preserve the topology of the graph in a distributed way and the connectivity between the vertices residing in the same partition. DPHV is designed to run on master-slaves architectures, as illustrated in Figure 6. DPHV algorithm is composed of two parts: coordinator() and partitioner().

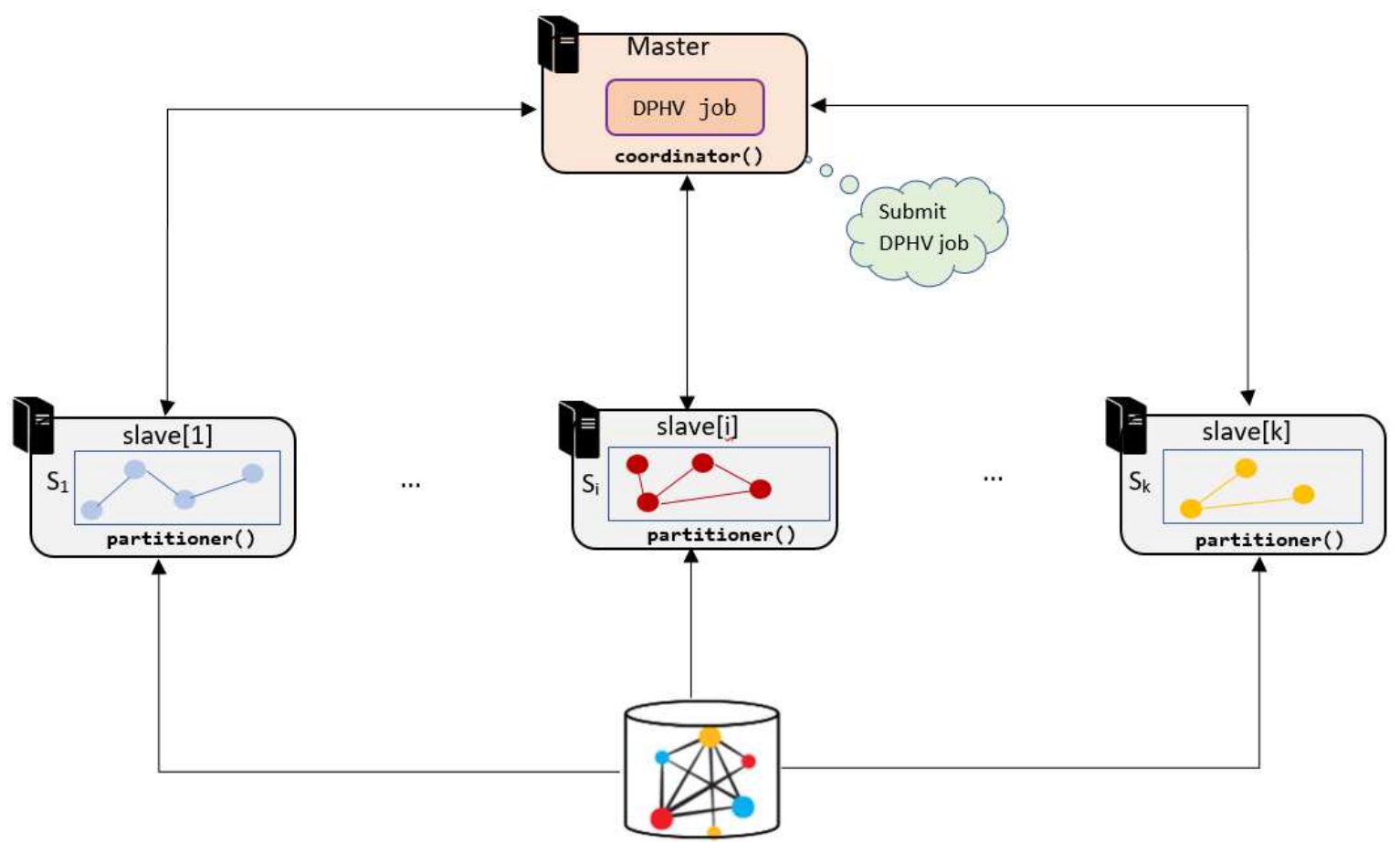

Fig. 6 Overview of the DPHV execution framework.

The parameters used in the DPHV heuristic pseudo-code are :

- $G=(V r, E d)$ : graph composed of set of vertex $V r$ and edge $E d$ such that $n=|V r|$ and $m=|E d|$.

- $k$ : number of sub-graphs of the graph $G r$ such that $k>2$. It is a hyperparameter which impacts the execution time and the result optimality.

- $G r_{i}^{\prime}$ : sub-graph $i$, such as $i \in \llbracket 1 ; k \rrbracket$ the weight of each partition is defined by $w\left(G r_{i}^{\prime}\right)$.

- slave[i]: slave node which hosts the partition $G r_{i}^{\prime}$.

- $s_{i}$ : vertex assigned to the partition $G r_{i}^{\prime}$, such as $s_{i} \in V r$. We denote $\operatorname{Vr}\left(s_{i}\right)$ the set of these adjacent vertices such that $d\left(s_{i}\right)$ is its degree.

- $M$ : contains all the vertices that have been assigned to one of the $k$ partitions.

- $C u t_{s_{i}}\left(G r_{i}^{\prime}\right)$ : number of cut-edges generated by the assignment of the vertex $s_{i}$ in the partition $G r_{i}^{\prime}$.

- $I n_{s_{i}}\left(G r_{i}^{\prime}\right)$ : number of induced edges generated by the assignment of $s_{i}$ to the sub-graph $G r_{i}^{\prime}$. 
- $f_{R}\left(G r_{i}^{\prime}, s_{i}\right)$ : ratio function of the number of induced edges compared to the number of cut-edges generated by the assignment of $s_{i}$ to $G r_{i}$. It is calculated as follows:

$$
f_{R}\left(G r_{i}^{\prime}, s_{i}\right)=\frac{I n_{s_{i}}\left(G r_{i}^{\prime}\right)}{\operatorname{Cut}_{s_{i}}\left(G r_{i}^{\prime}\right)}
$$

such as $C u t_{s_{i}}\left(G r_{i}^{\prime}\right)=1$ if there is no cut-edge generated by the assignment of the vertex $s_{i}$ to the sub-graph $G r_{i}^{\prime}$. Moreover, if no edge is generated inside the subgraph $G r_{i}^{\prime}$, then $I n_{s_{i}}\left(G r_{i}^{\prime}\right)=1$.

\subsection{The load balancer}

The coordinator () program is centralized on the master node, it is responsible of monitoring the state of the slave nodes and ensures that the weight of the partitions is equitably balanced across the cluster.

Algorithm 1 describes the process of coordinator(). Initially all nodes into the cluster are in the active state. So, at each iteration, the coordinator evaluates the value of the partition balance $B\left(P_{k}\right)$. If the balance constraints of the partition with respect to the acceptance error $\epsilon$ are not respected (see section 2) then, the coordinator checks at each iteration whether the weight $w\left(G r_{i}^{\prime}\right)$ of a subgraph $G r_{i}^{\prime}$ is not far from the average or is not too high compared to the other subgraphs. Then the coordinator puts it in inactive state via the haltNode(true, slave [i]) method.

When the subgraphs weights are balanced by comparison with the partition weight $G r_{i}^{\prime}$ then the coordinator puts the node slave $[i]$ in active state. Once all the vertices of the graph have been placed or marked, the coordinator deactivates all the slave nodes of the cluster and signals the end of the partitioning job.

\subsection{The distributed partitioning strategy}

Unlike the coordinator () which is centralized on the master node, partitioner () is decentralized on all the slave nodes of the cluster.

partitioner () processes and assigns each vertex before proceeding to the next one, it keeps in memory the current weight of its partition. In the event of q compromise, the following rules are used in the placement decision:

- If the majority of the neighbors of the current vertex are already in a subgraph $G r_{i}^{\prime}$, then the vertex will be added to this partition;

- If it has no subgraph in common, the subgraph with the most edges associated with this vertex will be chosen;

- If the vertex assignment generates the same placement ratio for all subgraphs, then the vertex will be assigned to the smallest subgraph $G r_{i}^{\prime}$ such that $w\left(G r_{i}^{\prime}\right)=\min \left\{w\left(G r_{1}^{\prime}\right), w\left(G r_{2}^{\prime}\right), \ldots, w\left(G r_{k}^{\prime}\right)\right\}$;

- Otherwise the vertex will be randomly assigned to one of the $k$ subgraphs.

It is important to emphasize that the graph provided as input is supported by the distributed storage system of Hadoop HDFS but without physically partitioning the graph. In this case, the size of the block file plays an important role because it defines the size of the sub-blocks making up each piece of data stored on the nodes of the cluster. This allows each node to have a global view on each block of the original graph and to ensure better information exchange between nodes. The data format of the graph supplied to enter is based upon the "Extended Property 


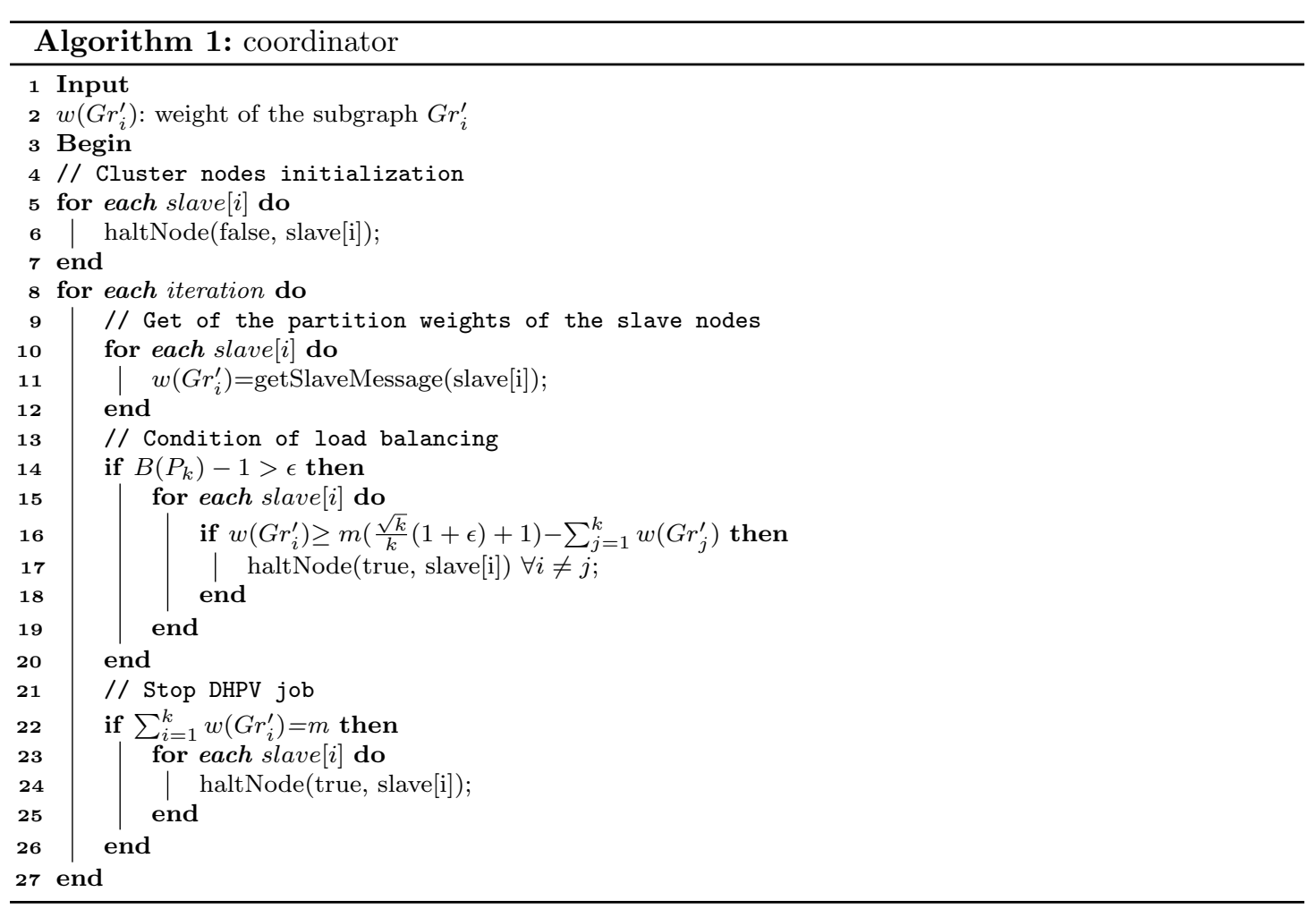

Graph Model" (EPGM) 43] model. Long before the partitioning phase, the vertices of the graph are ordered in ascending order of the degree of each vertex. This is done via a quick sorting by insertion operation whose execution time complexity is $O(n \log (n))$ et $O\left(n^{2}\right)$ in the worst case.

Algorithm 2 presents the pseudo-code of partitioner() program. Initially, each partition $G r_{i}^{\prime}$ is empty as well as the associated weight $w\left(G r_{i}^{\prime}\right)$ and the node slave $[i]$ receives a message from the master node notifying the start of the partitioning task. At each iteration, as long as the node slave $[i]$ does not receive a message signaling the end of the job, then for each $k$ unmarked vertices, we evaluate the ratio $f_{R}\left(G r_{i}^{\prime}, s_{i}\right)$ of the number of induced edges generated by the placement of this vertex $s_{i}$ in the partition $G r_{i}^{\prime}$ compared to the number of cut-edges generated by the placement of the vertex $s_{i}$. Then the vertex $s_{i}^{*}$ having the maximum value of $f_{R}\left(G r_{i}^{\prime}, s_{i}^{*}\right)$ is chosen. If two slave nodes slave $[i]$ and slave $[j]$ whose vertices $s_{i}^{*}$ and $s_{j}^{*}$ are promising and if $w\left(G r_{j}^{\prime}\right) \geq w\left(G r_{i}^{\prime}\right)$ then the vertex $s_{i}^{*}$ will be placed in the partition $G r_{i}^{\prime}$ while the vertex $s_{j}^{*}$ will be replaced by the vertex $s_{j-1}$ and placed in the partition $G r_{j}^{\prime}$. Each of the $k$ vertices placed is marked. Subsequently we add all incident edges to vertex $s_{i}$ as well as the cut-edges generated by the assignment of $s_{i}$. Finally, the slave node slave $[i]$ communicates by message the new value of the weight of its partition to the master node. It is important to emphasize that the partition task is parallelized according to BSP (Bulk Synchronous Parallel) paradigm [41. So when a node finishes placing a vertex, it waits until the rest of nodes finish their job. Thus, the time complexity of each node is $O\left(\frac{\left|V r\left(s_{i}\right)\right| n^{2}}{k} \log (k)\right)$. 


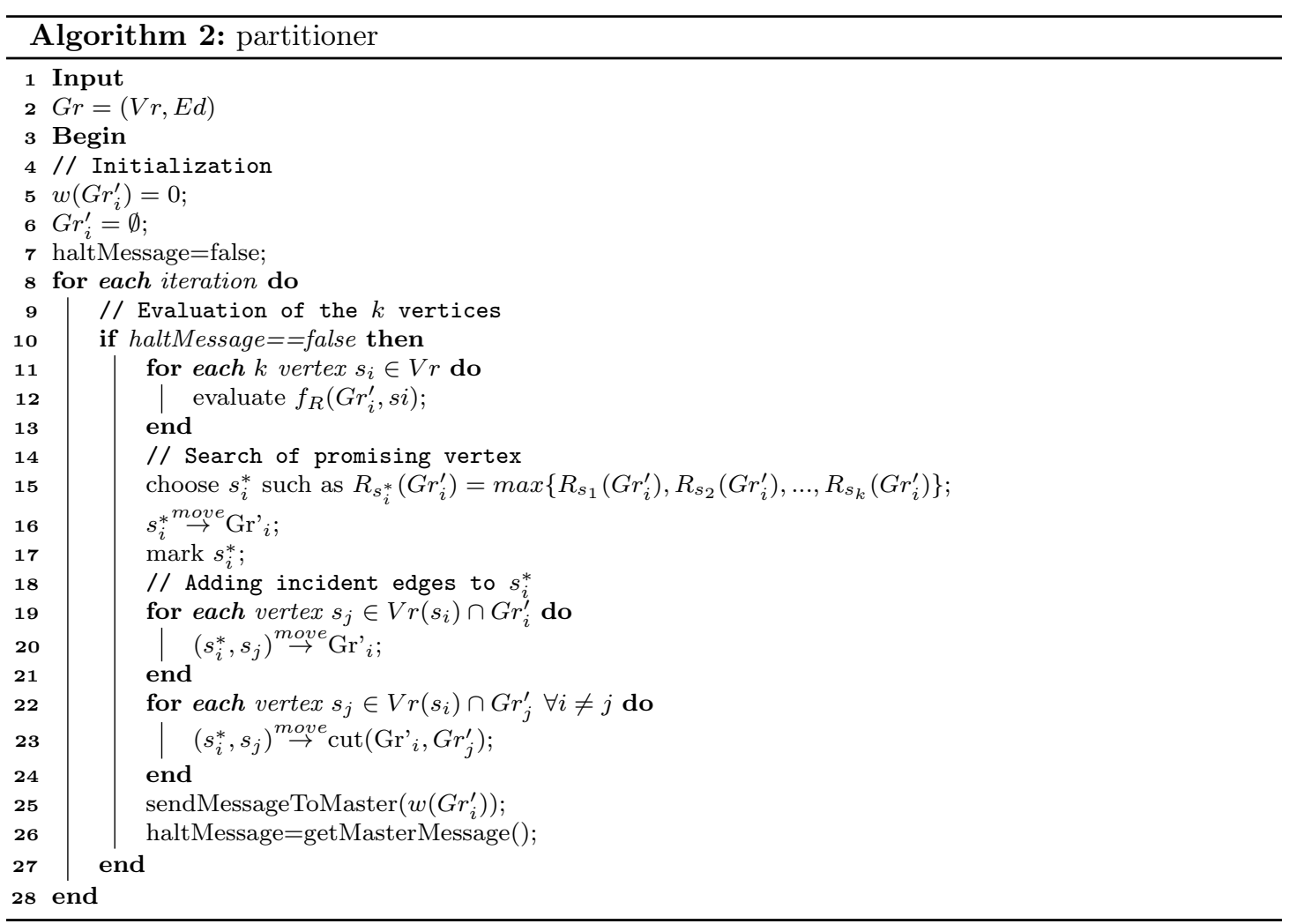

\section{Results and Discussions}

\subsection{Illustration of DPHV algorithm}

We highlight a simple illustration of DPHV algorithm for $k=2$. Let $G r=(V r, E d)$ be a graph composed of 7 vertices and 10 edges which we want to partition into 2 sets $G r_{1}^{\prime}$ and $G r_{2}^{\prime}$. It is assumed that the cluster used is set up of 2 slave nodes which perform the partitioning task while the master node supervises the DPHV job.

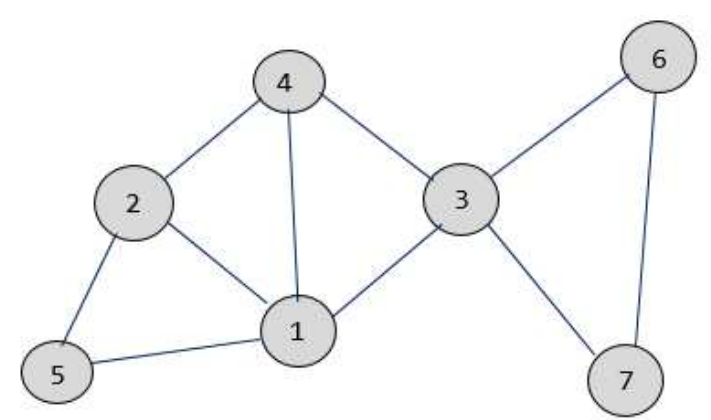

Fig. 7 Graph $G r=(V r, E d)$ such as $|V r|=7$ et $|E d|=10$ 


\subsubsection{The pre-processing phase}

This phase consists of ordering the 7 vertices of the graph in descending order of their respective degrees. Table 2 presents the vertices as well as the degree of each vertex. Initially the two partitions are empty and their respective weights are $w\left(G r_{1}^{\prime}\right)=w\left(G r_{2}^{\prime}\right)=0$.

Table 1 Degree of vertices of the graph.

\begin{tabular}{|l|l|l|l|l|l|l|l|}
\hline$s_{i}$ & 1 & 3 & 2 & 4 & 7 & 5 & 6 \\
\hline$d\left(s_{i}\right)$ & 5 & 4 & 3 & 3 & 3 & 2 & 2 \\
\hline
\end{tabular}

\subsubsection{The partitioning phase}

Iteration 1: initially each slave node randomly selects the first $k=2$ vertices $\left\{s_{1}, s_{3}\right\}$ not marked. In this example, vertex $s_{1}$ is assigned to the partition $G r_{1}^{\prime}$ while vertex $s_{3}$ is assigned to the partition $G r_{2}^{\prime}$. Then the internal edges and cut-edges are added. Once this task is completed, the two vertices will be marked.

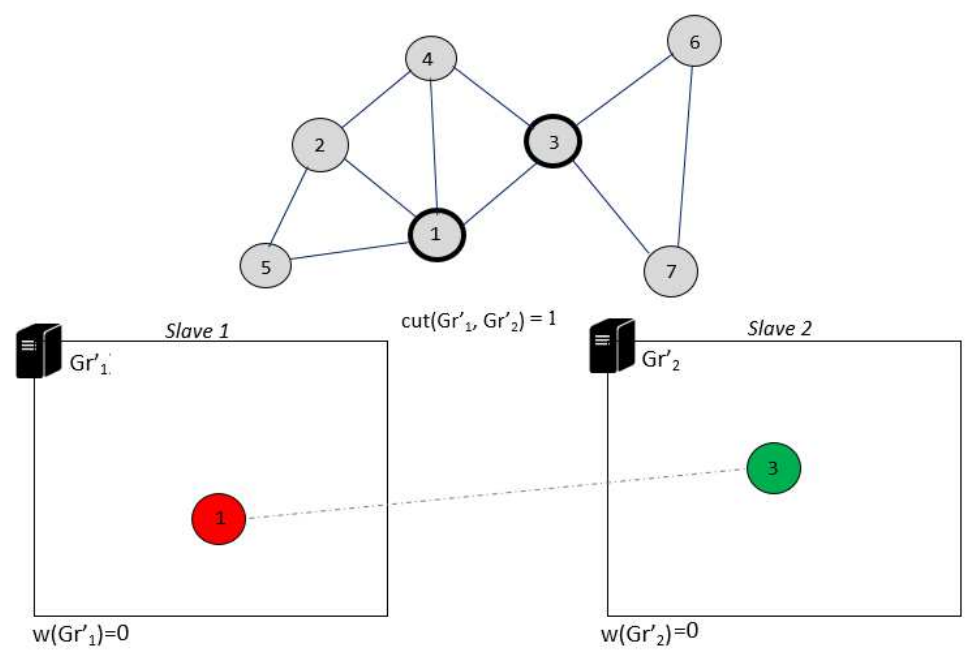

Fig. 8 Iteration 1 of DPHV execution.

Iteration 2: then the other two unmarked vertices $\left\{s_{2}, s_{4}\right\}$ are selected. Thus, in parallel the two slave nodes evaluate the following operations:

$s_{2} \stackrel{\text { move }}{\rightarrow}$ slave[1] $\Longrightarrow f_{R}\left(G r_{1}^{\prime}, s_{2}\right)=1\left(C u t_{s_{2}}\left(G r_{1}^{\prime}\right)=1, \operatorname{In}_{s_{2}}\left(G r_{1}^{\prime}\right)=1\right)$

$s_{4} \stackrel{\text { move }}{\rightarrow}$ slave[1] $\Longrightarrow f_{R}\left(G r_{1}^{\prime}, s_{4}\right)=1,\left(C u t_{s_{4}}\left(G r_{1}^{\prime}\right)=1, I_{s_{4}}\left(G r_{1}^{\prime}\right)=1\right)$

$s_{2} \stackrel{\text { move }}{\rightarrow}$ slave $[2] \Longrightarrow f_{R}\left(G r_{2}^{\prime}, s_{2}\right)=1,\left(C u t_{s_{2}}\left(G r_{2}^{\prime}\right)=1, I_{s_{2}}\left(G r_{2}^{\prime}\right)=1\right)$

$s_{4} \stackrel{\text { move }}{\rightarrow}$ slave $[2] \Longrightarrow f_{R}\left(G r_{2}^{\prime}, s_{4}\right)=1\left(C u t_{s_{4}}\left(G r_{2}^{\prime}\right)=1, \operatorname{In}_{s_{4}}\left(G r_{2}^{\prime}\right)=1\right)$

The vertices $s_{2}$ and $s_{4}$ are marked and placed respectively in the partitions $G r_{1}^{\prime}$ and $G r_{2}^{\prime}$.

Iteration 3: We repeat the same operations again by selecting the $k=2$ unmarked vertices $\left\{s_{5}, s_{7}\right\}$. Each slave node then evaluates the best placement:

$s_{7} \stackrel{\text { move }}{\rightarrow}$ slave $[1] \Longrightarrow f_{R}\left(G r_{1}^{\prime}, s_{7}\right)=1\left(C u t_{s_{7}}\left(G r_{1}^{\prime}\right)=1, I n_{s_{7}}\left(G r_{1}^{\prime}\right)=1\right)$ 

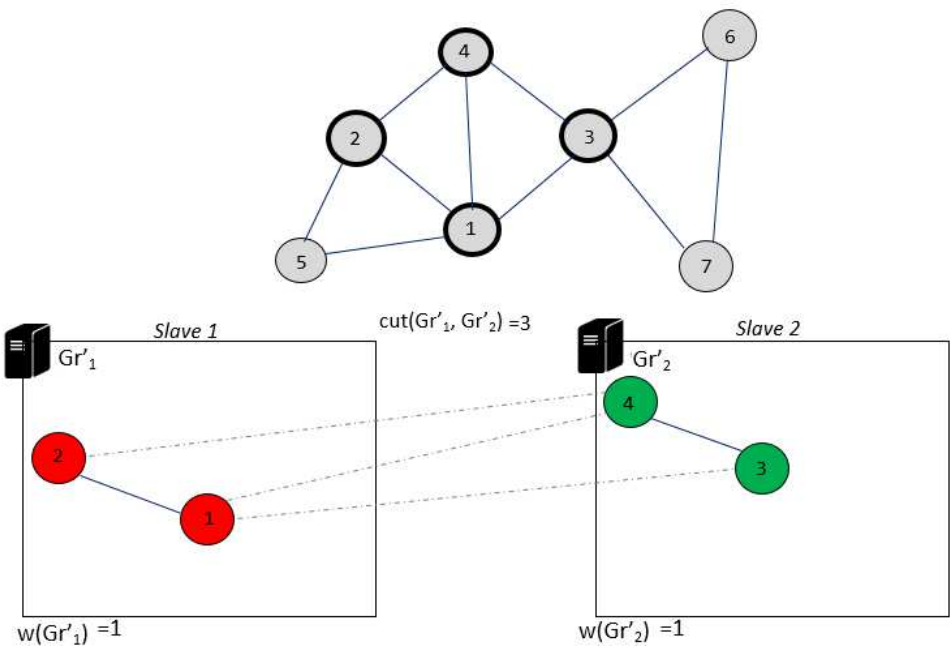

Fig. 9 Iteration 2 of DPHV execution.

$s_{5} \stackrel{\text { move }}{\rightarrow}$ slave[1] $\Longrightarrow f_{R}\left(G r_{1}^{\prime}, s_{5}\right)=2\left(C u t_{s_{5}}\left(G r_{1}^{\prime}\right)=1, I n_{s_{5}}\left(G r_{1}^{\prime}\right)=2\right)$

$s_{7} \stackrel{\text { move }}{\rightarrow}$ slave $[2] \Longrightarrow f_{R}\left(G r_{2}^{\prime}, s_{7}\right)=1\left(C u t_{s_{7}}\left(G r_{2}^{\prime}\right)=1, I n_{s_{7}}\left(G r_{2}^{\prime}\right)=1\right)$

$s_{5} \stackrel{\text { move }}{\rightarrow}$ slave $[2] \Longrightarrow f_{R}\left(G r_{2}^{\prime}, s_{5}\right)=1\left(C u t_{s_{5}}\left(G r_{2}^{\prime}\right)=1, I_{s_{5}}\left(G r_{2}^{\prime}\right)=1\right)$

The vertices $s_{5}$ and $s_{7}$ are marked and placed respectively in the partitions $G r_{1}^{\prime}$ and $G r_{2}^{\prime}$.

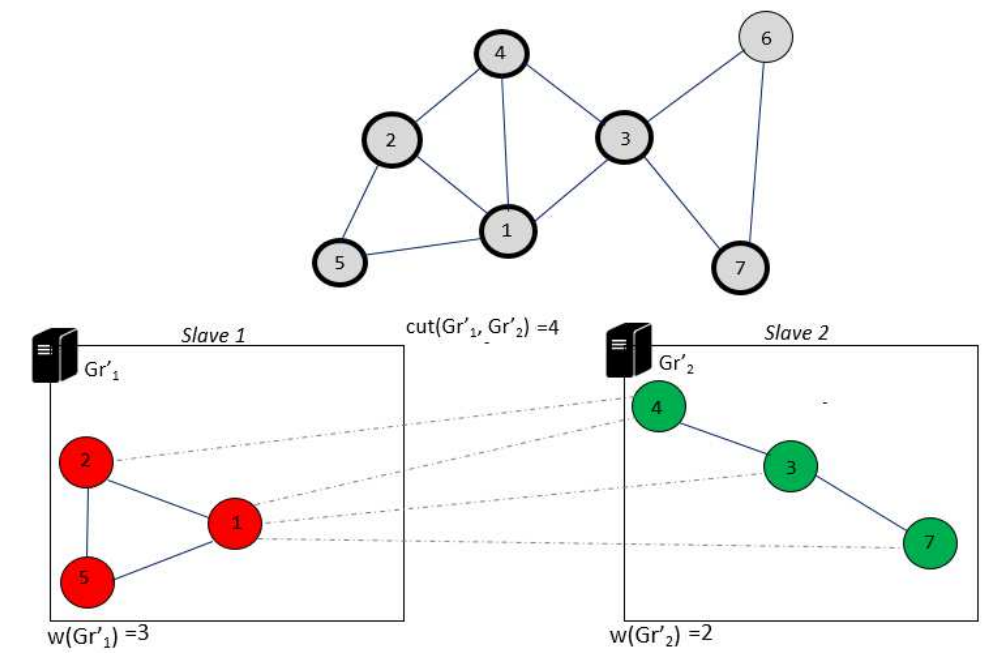

Fig. 10 Iteration 3 of DPHV execution. 
Iteration 4: Finally, in the last part, the last unmarked vertex $s_{6}$ is evaluated in parallel to determine the placement which generates less cut-edges:

$$
\begin{aligned}
& s_{6} \underset{\text { move }}{\rightarrow} \text { slave[1] } \Longrightarrow f_{R}\left(G r_{1}^{\prime}, s_{6}\right)=1\left(C u t_{6}\left(G r_{1}^{\prime}\right)=2, I_{s_{6}}\left(G r_{1}^{\prime}\right)=0\right) \\
& s_{6} \stackrel{\text { move }}{\rightarrow} \text { slave }[2] \Longrightarrow f_{R}\left(G r_{2}^{\prime}, s_{6}\right)=2\left(C u t_{s_{6}}\left(G r_{2}^{\prime}\right)=1, I n_{s_{6}}\left(G r_{2}^{\prime}\right)=2\right)
\end{aligned}
$$

The vertex $s_{6}$ is placed in the partition $G r_{2}^{\prime}$ because it generates less cut-edges. The algorithm stops because all vertices are marked. The solution obtained is one of the solutions that DPHV can generate. Another solution would be to place the vertex $s_{4}$ in the partition $G r_{1}^{\prime}$, this will reduce the number of cut-edges to $w\left(G r_{1}^{\prime}, G r_{2}^{\prime}\right)=3$.

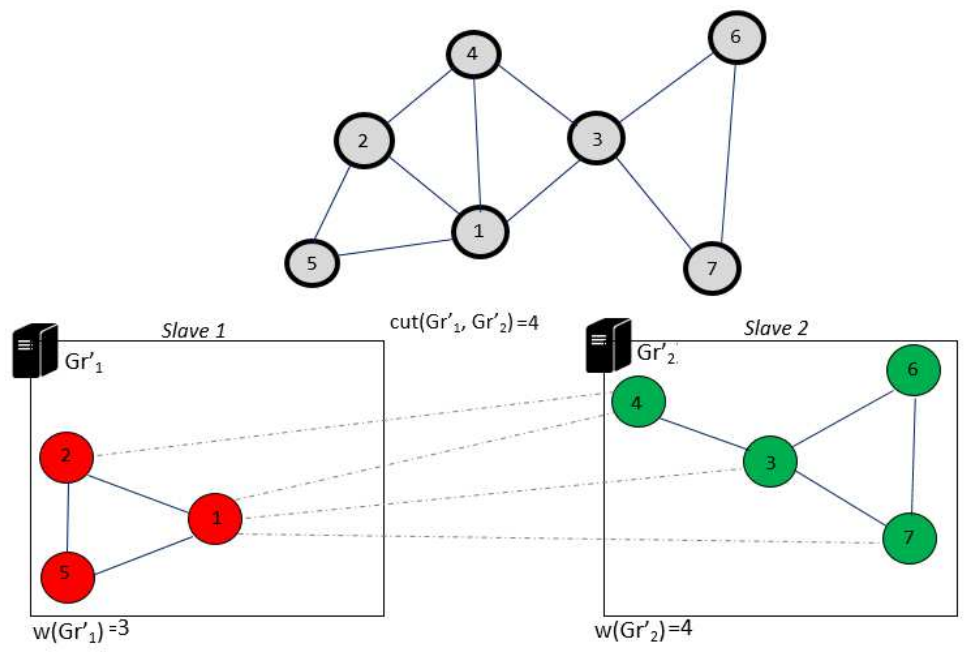

Fig. 11 Iteration 4 of DPHV execution.

The main drawback of DPHV is that it does not optimize the solution obtained in order to get as close as possible to the optimal. On the other hand, it allows the placement of a dynamic graph according to the same paradigm. DPHV is even faster when the number of partitions is small. When the number of partitions increases, the master node performs more operations because it will be necessary to regulate the weight of each partition so as to remain within the limits of acceptance constraints. When for example $l$ slave nodes go into inactive state, the problem of $k$-partition automatically switches to a problem of $k^{\prime}$-partition with $k^{\prime}=k-l$. In addition, unlike other partitioning strategies which perform random placement of vertices or edges, DPHV is completely de-randomized, which means that regardless of the topology of the graph, the algorithm cannot fall into a local optimal.

\subsection{Test environment and dataset}

The experimental tests were carried out on the Grid'5000 ${ }^{1}$ an open platform for cloud computing. It is dedicated exclusively to experimental tests involving high performance computations on parallel and distributed systems. This platform already incorporates big data platforms like Spark [15, GraphX [33] and Hadoop [14. The cluster allocated for the tests contains 10 nodes configured in a homogeneous manner (see Figure 12). The experimental tests were also carried

\footnotetext{
1 https://www.grid5000.fr
} 
out by changing the number of nodes. Each node is equipped with 240 GB SSD + 480 GB SSD + 4.0 TB HDD, 140 GB of RAM, 10 Gbps + 100 Gbps of Omni-Path Ethernet cables and a 2 x Intel Xeon Gold 6130 (16 cores/ CPU). We adopted Ganglia 42 for monitoring the cluster's performance.

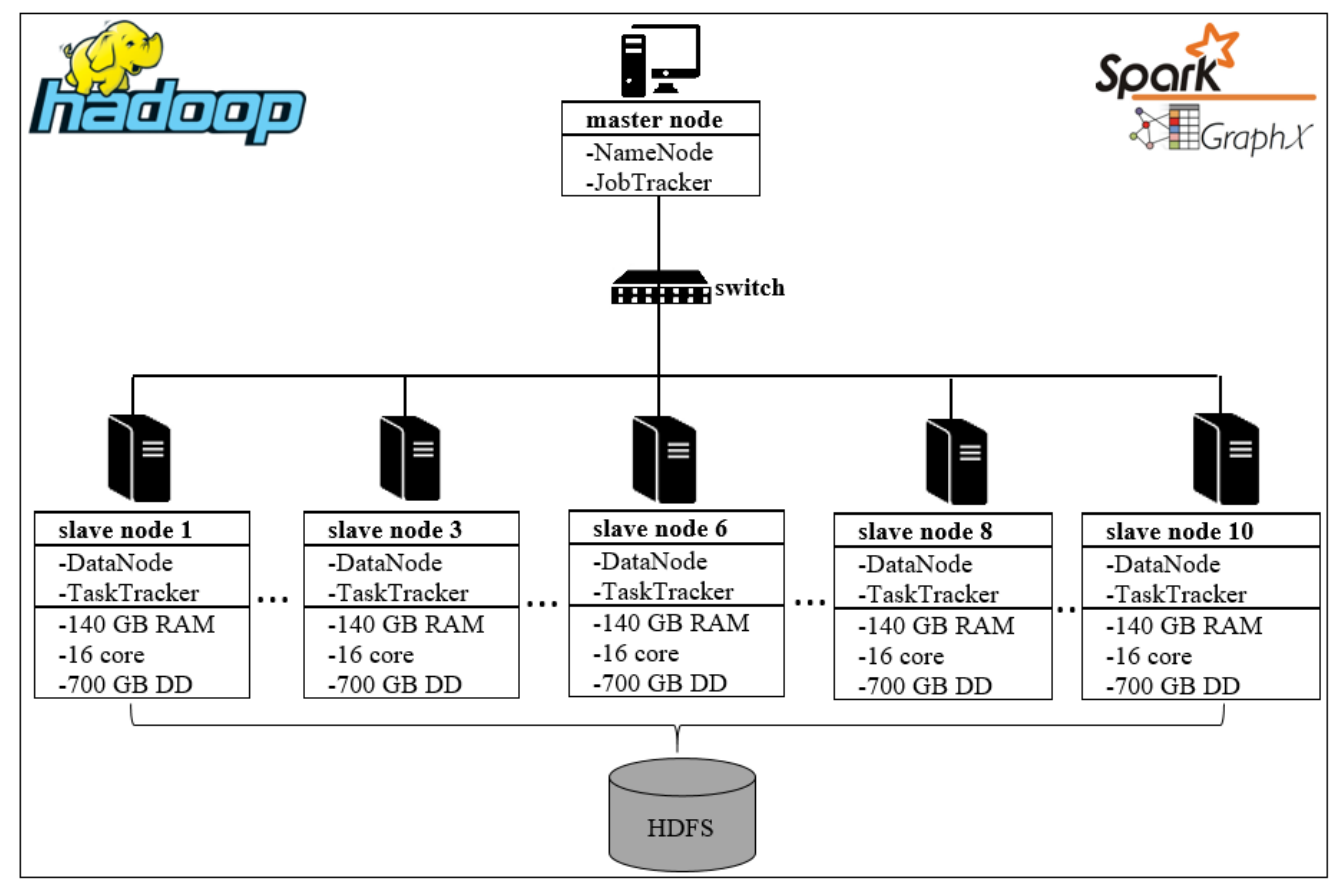

Fig. 12 Test environment [15]14].

We used benchmark data extracted from SNAP 2 a large network dataset collection. To highlight the applicability and the performances of our heuristic, we used two categories of datasets. The first category represents deterministic finite automata with explosion of states. These data were extracted from the set of different conformance test models of various complex systems ${ }^{3}$ These datasets correspond to the finite behavioral models of the test tools piloted by formal verification models for the performance of conventional test tasks: selection of test cases, prioritization, mutation tests, etc. The second category of datasets used represents the road network of Morocco collected from the OpenStreetMap (OSM) spatial database ${ }^{4}$. It contains points, different types of roads and lanes between two points of interest. Each entity of the road network contains tags nested in each of these objects. The graph of the road network covers all types of road, including local roads. It contains directed and weighted edges to estimate distances/time of travel.

Table 2 presents the characteristics of the graphs extracted from the datasets. For each dataset, we present the number of vertices $|V r|$, the number of edges $|E d|$, the diameter of the graph $D$ and the clustering coefficient $A C C$.

\footnotetext{
2 https://snap.stanford.edu/

3 https://projects.info.unamur.be/vibes/mutants-equiv.html

4 http://download.geofabrik.de/
} 
Table 2 Datasets

\begin{tabular}{|l|l|l|l|l|l|}
\hline Designation & Type & $|\mathbf{V r}|$ & $|\mathbf{E d}|$ & ACC & D \\
\hline biosnap & undirected & 1018524 & 24735503 & 0.42 & 15 \\
\hline twitter & directed & 81306 & 1768149 & 0.5653 & 7 \\
\hline usroad & directed & 126146 & 161950 & 0.0145 & 617 \\
\hline email & undirected & 36692 & 183831 & 0.4970 & 11 \\
\hline astro & undirected & 18772 & 198110 & 0.6306 & 14 \\
\hline ageRRN & directed & 860000 & 2360000 & 56 & 0.4970 \\
\hline cptTRM & directed & 1070000 & 6000000 & 70 & 0.6235 \\
\hline elsaRR & directed & 1508000 & 9000000 & 92 & 0.5653 \\
\hline osmMA & directed & 4526700 & 12670000 & 412 & 0.0136 \\
\hline
\end{tabular}

\subsection{Complexity of graph partitioning algorithms}

Vertex-partition [5, Edge-partition 21, Spectral 25, Kernighan-Lin 27, Metis 30] and Greedy 23 methods were implemented in python 3.4. DFED [21] method was implemented in Java 8.2 for MapReduce [14] version and Scala 2.12 for Spark in-memory version [44. JA-BE-JA 24] method was also written in Scala 2.12. Each network is partitioned under $k=25$ partitions. The algorithms have been executed ten times to get an average parameter values: the runtime, the cost communication and the load balancing. A partitioning method is considered effective if it is both fast and results in balanced partitions with fewer cut-edges. Figure 13 illustrates the performance of different techniques according to the hyper parameters. The methods that can satisfy these three constraints are: Vertex-partition [5], Edge-partition [21, Spectral 25], Metis [30, Kernighan-Lin [27] and Fennel [34. The performances of JA-BE-JA [24], DFED [21, Greedy [23] vary according to the graph topology. For example, Greedy [23] is very fast in terms of velocity but generates a large number of cut-edges and the partition is not balanced.

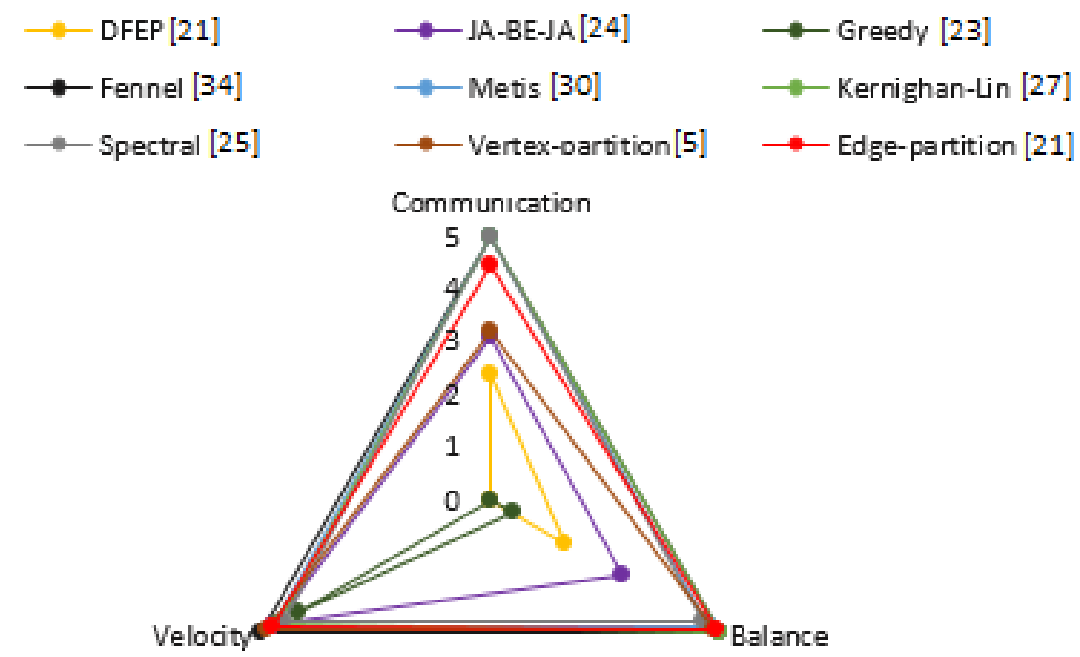

Fig. 13 Computational complexity of graph partitioning methods

Figure 14 introduces for the constraints satisfaction rate of each graph partitioning method. The performance of each algorithm is the result of crossing the three evaluation parameters. The rate of the maximum performance obtained varies from 1 to $100 \%$. A rate close to $100 \%$ means that all of the constraints are satisfied. 

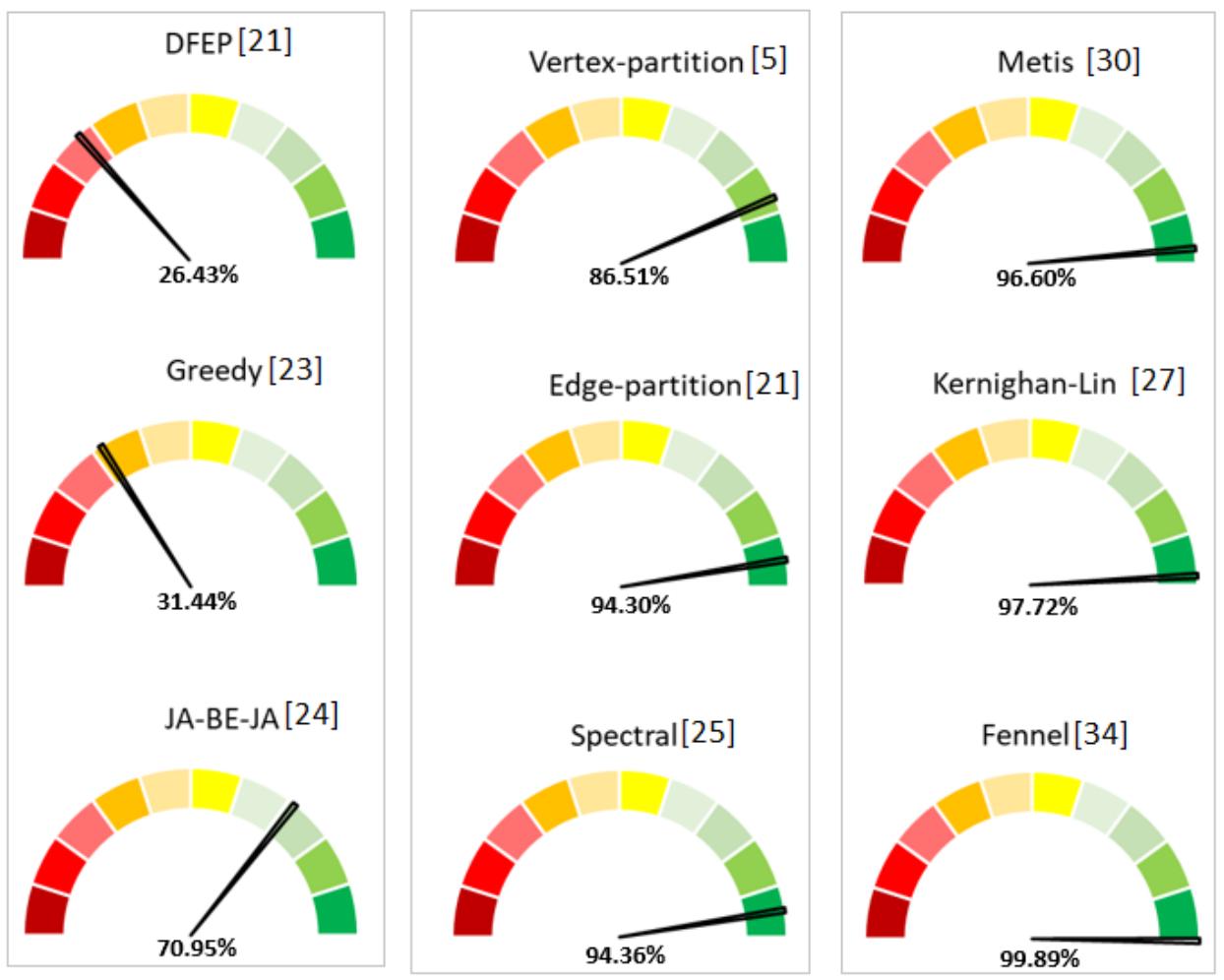

Fig. 14 Constraints satisfaction rate.

We observe that Fennel [34, Metis [30, Kernighan-Lin 27] and Spectral 25] have a satisfaction rate that ranges between $94 \%$ and approximately $100 \%$. This highlights the previous results analysis. DFED 21] and Greedy 23] have a lower constraints satisfaction rate. However, it is important to emphasize that this rate can vary depending on the graph topology and the cluster configuration. A performance rate below the average does not necessarily imply that the method is ineffective. There are a number of trade offs in choosing an appropriate graph partitioning method [8]. This involves in-depth reflection in the pre-processing phase.

\section{Computational complexity of DPHV}

The study of the complexity of DPHV was carried out on osmMA dataset in order to better understand its complexity of our algorithm. We opted for this dataset because it is a complex network which puts all the evaluation parameters of our approach into competition. We also analyze the behavior of DPHV with respect to the variation in the number of partitions $k$. Then we make a comparison with benchmark models of existing distributed partitioning algorithms. The evaluation parameters highlighted are 1) the time complexity; 2) communication costs of the cluster, 3) the load balance and 4) the connectivity of the sub-graphs induced in each partition [5,6]. DPHV program was written in Java and the different Jobs run on JVM 1.8.

Figure 15 shows the behavior of DPHV compared to the number of partitions. Note that the variation of the number of partitions has a great impact on the behavior of DPHV. When the number of partitions increases, the algorithm tends to run slowly, which is quite logical since the 
time taken to partition a graph into $(k+1)$ partitions is significant than that of a $k$ partitions. In addition, for a high $k$, DPHV will spend more time evaluating a large number of vertices at each iteration. This greatly influences the time complexity but allows obtaining a better partition which minimizes cut-edges. We also note that whatever the variation of the number of partitions, the percentage of cut-edges varies between $20 \%$ and $30 \%$. This is remarkable because it demonstrates that DPHV manages to stabilize the evolution of cut-edges, thus making it possible to reduce or control the costs of communication.

The standard deviation in Figure 15 (c) oscillates between 0.9 and 0.98 , this value is very close to 1 whatever the number of partitions. We can conclude that DPHV balances the partition weights so that the constraint $B\left(P_{k}\right) \leq(1+\epsilon)$ is respected. This aspect is very important because it ensures good parallelism [8, 40] with workloads distributed evenly on each node of the cluster. This reduces the latency the time spent in synchronization tasks.

Figure 15 (d) shows that the standard deviation oscillates between 0.6 and 0.8 , this means that the connectivity of each subgraphs induced in each partition remains close from that of the original graph. In addition, our heuristic is the first to propose partitions whose vertices induced in each partition are strongly connected.

DPHV is able to partition large-scale graphs in a parallel and distributed architecture, all while preserving the graph topology as much as possible. While optimizing the number of cut-edges in order to minimize the communication costs. In addition, DPHV is scalable and supports the large-scale graph.

(a) Runtime

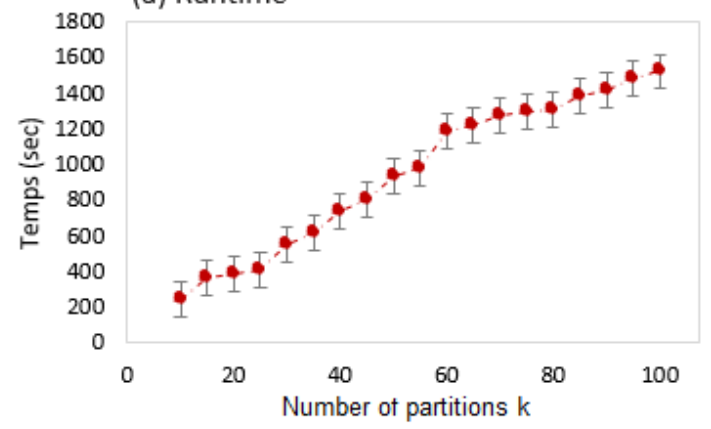

(c) Balance

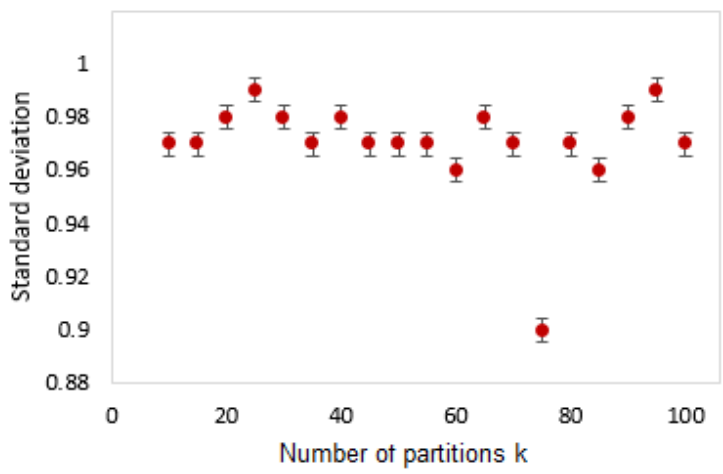

(b) Cross-edges between partitions

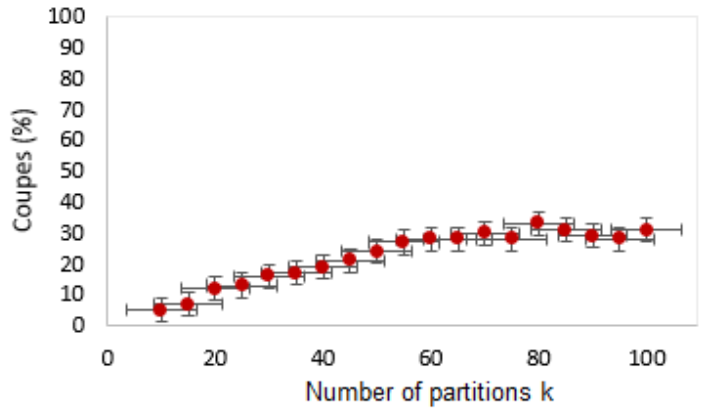

(d) Connectivity of induced sub-graphs

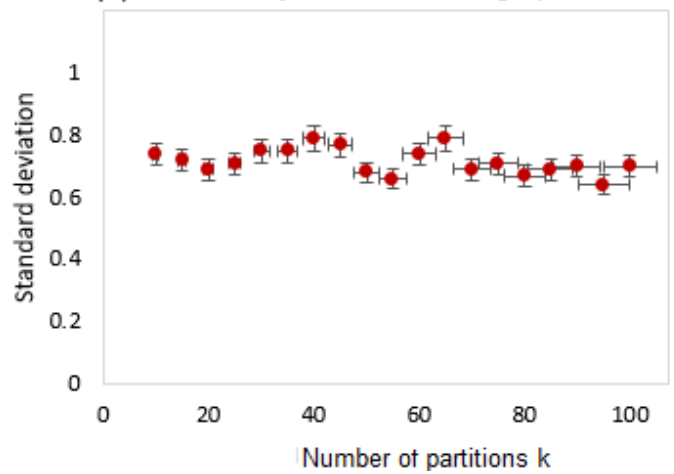

Fig. 15 DPHV behavior based on the number of partition $k$. 
We compare our approach with others parallel and distributed algorithms: DFEP [21, JA-BE-JA 24 and Greedy 23. Figure 16 shows the experimental results of graph algorithms on the datasets. In terms of velocity, Greedy 23 and DFED 21 outperform the performance of our algorithm. But DPHV presents better results of cut-edges compared to Greedy and DFED. The cut-edges with JA-BE-JA [24] are much better than ours.

In terms of partition balancing, our approach presents the best results compared to other algorithms. Also, our algorithm presents partitions whose sub-graphs induced in each partition are strongly connected.
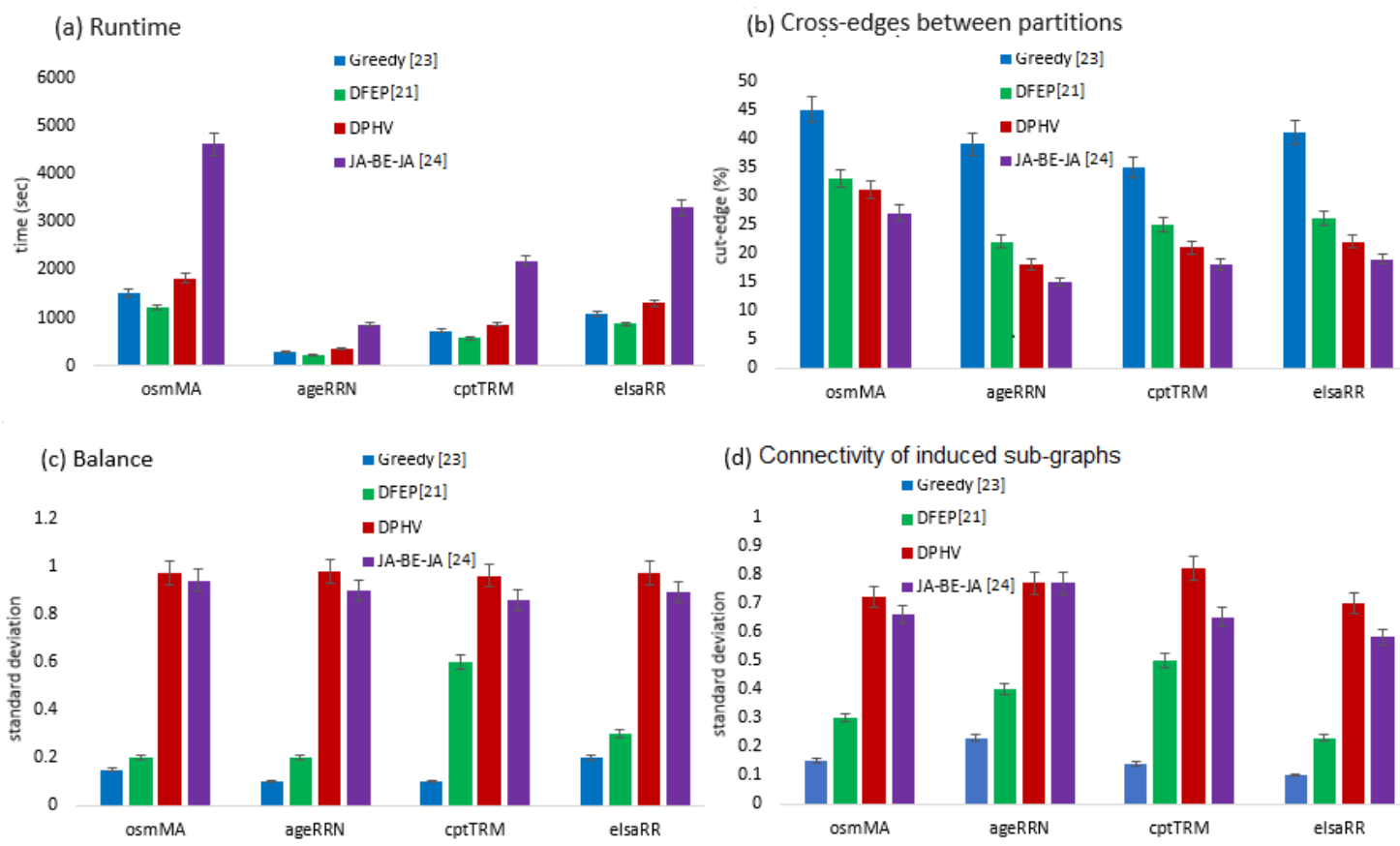

(d) Connectivity of induced sub-graphs

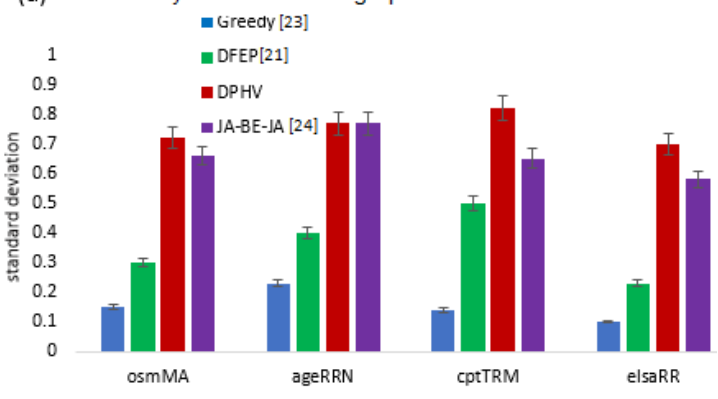

Fig. 16 Comparison between DPHV, DFEP [21], JA-BE-JA [24] and Greedy [23] (k=100)

Table 3 shows the gain ratio of our partitioning method compared to other partitioning techniques.

Table 3 DPHV performance ratio compared to DFEP [21], JA-BE-JA 24] and Greedy 23]

\begin{tabular}{|c|c|c|c|}
\cline { 2 - 4 } \multicolumn{1}{c|}{} & DPHV vs. Greedy $[23]$ & DPHV vs. DFEP $[21]$ & DPHV vs. JA-BE-JA $[24]$ \\
\hline Time & -1.2 & -1.5 & $\mathbf{+ 2 . 5 3}$ \\
\hline Cut-edges & $\mathbf{+ 1 . 7 4}$ & $\mathbf{+ 1 . 1 5}$ & -0.86 \\
\hline Balance & $\mathbf{+ 7 . 0 8}$ & $\mathbf{+ 2 . 9 8}$ & $\mathbf{+ 1 . 0 8}$ \\
\hline Connectivity & $\mathbf{+ 4 . 8 5}$ & $\mathbf{+ 2 . 1}$ & $\mathbf{+ 1 . 1 3}$ \\
\hline
\end{tabular}

In terms of velocity, DPHV is 1.2 times slower than Greedy and 1.5 times slower than DFEP 21]. But it is 2.53 times faster than JA-BE-JA [24. The communication costs (cut-edges) proposed by our approach are 1.74 times reduced compared to that of Greedy [23] and 1.15 times reduced compared to DFEP [21]. On the other hand, the cut-edges of JA-BE-JA [24] optimize these cuts by 0.86 times than that of our algorithm. In terms of load balancing, our 
results are remarkable compared to the others. Our technique offers partitions whose weights are 7 times more balanced compared to Greedy [23] and 3 times more balanced compared to DFEP [21. Similarly, in terms of connectivity, the partitions proposed by DPHV are strongly connected, this means that we maintain the topology of the original graph.

Besides, our algorithm outperforms all the distributed algorithms in terms of connectivity of the induced sub-graphs and offers the best performance. Our results are almost 5 times better than those of Greedy [23, 2 times better than those proposed by DFEP [21] and finally 1.13 times better than the connectivity of the induced sub-graphs resulting from the partitioning of JA-BE-JA [24].

\subsection{Discussions}

Despite the fact that DPHV is efficient compared to other distributed algorithms [21,24,23, there are some limitations regarding the conceptual model, the programming paradigm and the applicability:

1. Node storage capacity [15]: DPHV is based on the Spark architecture and makes extensive use of certain functions of the Spark API. These functions are optimized for in-memory computations. In the case of big graphs, it becomes expensive to store the graph on RAM memory. So to maintain a good performance of our algorithm, we will have to allocate additional RAM memory.

2. Hardware failure [45]: DPHV partitioning task is completely decentralized to the slave nodes of the cluster. In the conceptual model of DPHV, when a node fails or is unavailable, the partitioning switches from a k-partition to a $\left(k-k^{\prime}\right)$-partition where $k^{\prime}$ is the number of unavailable nodes. This therefore affects the weight of the sub-graphs contained in the unavailable nodes. Therefore, balancing the weights of the partitions cannot be guaranteed. Also, the cut-edges will be affected, resulting in a considerable communication cost.

3. Physical partitioning of graphs [14]: The partitioning logic of DPHV is based on the degree of the vertices in order to have balanced partitions. In the definition of a big graph, the vertices and edges store a large amount of data. Unfortunately, this is not taken into account in the DPHV partitioning logic. Therefore, despite the fact that the weights of the sub-graphs are balanced, they do not store the same amount of information.

In terms of complexity, our approach has advantages over DFEP 21. Because in each iteration, it exchanges fewer vertices than DFEP [21. As a result, DPHV optimizes the use of hardware resources such as ram memory, CPU and network processor.

Compared to our approach, JA-BE-JA [24 provides more optimal partitions because it is based on simulated annealing [39]. But very expensive in terms of time complexity and hardware resources.

Similarly, Greedy 23] outperforms our algorithm, but it is adapted for high-performance computing with single machine. This is very expensive in terms of hardware resources because it requires a very costly supercomputer [5].

\section{Conclusion and future work}

In this paper, we have proposed a concrete formalism of the k-partition problem on big graphs. Moreover, we proposed a comparative study and a roadmap of partitioning algorithms. We introduced DPHV, a distributed k-partition algorithm based on a master-slaves architecture. In 
terms of velocity, DPHV is very fast and efficiently partitions a big graph into k sub-graphs of nearly similar weight while optimizing the number of cut-edges of the partition. DPHV also retains the topology of the original graph in a distributed architecture. The conceptual model of our framework is based on a coordinator and a set of partitioners. Experimental results have shown that our partitioning technique guarantees two fundamental properties : (1) the balancing of partition weights and (2) the preservation of the original graph topology in a distributed environment.

For future work, we are interested in expanding the scope of this work in the fight against covid-19. In particular by applying DPHV for the partitioning of large-scale community network, we can perform the propagation analysis and prediction of the COVID-19 by using all-shortest paths algorithms [4. In addition, we are interested in proposing an extended version of the DPHV algorithm which sorts the vertices of the graph in such a way that the data contained in the vertices are consistent when Hadoop [45] physically splits the graph file.

\section{List of abbreviations}

DPHV: Distributed Placement of Hub-Vertices

DFEP: Distributed Funding-Edge Partitioning

IoT: Internet of Things

CAP: Consistency Availability Partition

EPGM: Extended Property Graph Model

CRUD: Create Read Update Delete

NoSQL: Not only SQL

HDFS: Hadoop Distributed File System

SNAP: Stanford Network Analysis Platform

BSP : Bulk Synchronous Parallel

JOSM: Java Open Street Map

OSM: Open Street Map

\section{Availability of data and materials}

The data used for this study are available at :

https://snap.stanford.edu/data/index.html

https://projects.info. unamur.be/vibes/mutants-equiv.html

http://download.geofabrik.de/

\section{Competing interests}

The authors declare that they have no competing interests

\section{Ethics approval and consent to participate}

Not applicable. 


\section{Consent for publication}

Not applicable.

\section{Funding}

Not applicable.

\section{Author's contributions}

All mentioned authors contribute in the elaboration of the article. All authors read and approved the final manuscript.

\section{Authors' information}

"Dr Wilfried Yves Hamilton Adoni is a Researcher Professor of Computer Science at Interntional University of Casablanca. He is in the final step of this PhD in Big Data and High Performance Computing at Hassan II University of Casablanca, Morocco. He received the M.E. degree in Operational Research and System Optimization from Hassan II University of Casablanca, Morocco in 2014. He received the B.S. degree in Computer Science from Hassan II University of Casablanca, Morocco in 2012. His research interests include Big Data, Graph database, Smart transportation, Machine Learning and Deep Learning. He joined the elite group of young Big Data Specialist with IBM BigInsights V2.1 in 2015. Dr Wilfried Adoni is the corresponding author and can be contacted at: adoniwilfried@gmail.com / wilfried.adoni09@etude.univcasa.ma "

"Dr Tarik Nahhal obtained his HDR (Ability to Conduct Researches) in Computer Science from the Faculty of Science at Hassan II University of Casablanca. He holds a PhD in Hybrid System and Artificial Intelligence from the Joseph Fourier University, Grenoble I, France. His research interests include Hybrid System, Cloud Computing, Big Data, NoSQL Database, IoT and Artificial Intelligence. He has animated several Big Data conferences and has published several research articles in peer-reviewed international journals and conferences. He is Big Data specialist with IBM Big Data certification. His current research interests focus on high problem related to Big Data complexity. Dr Tarik Nahhal can be contacted at: t.nahhal@fsac.ac.ma ."

"Dr Moez Krichen obtained his HDR (Ability to Conduct Researches) in Computer Science from the University of Sfax (Sfax, Tunisia) in 2018. He Obtained his PhD in Computer Science in 2007. He is currently an Assistant Professor at the National School of Engineers of Sfax and member of the Research Laboratory on Development and Control of Distributed Applications REDCAD (Sfax, Tunisia). His main Research Interest is Model-Based Conformance/Load/Security Testing Methodologies for Real-Time, Distributed and Dynamically Adaptable Systems. Moreover he works on applying Formal Methods to several Modern Technologies like Smart Cities,Internet of Things (IoT), Smart Vehicles, Drones, Healthcare Systems, etc.Currently he is also working on Formal Aspects related to Deep Learning,Data Mining, Block-chain, Smart Contracts and Optimization. Dr Moez Krichen can be contacted at: moez.krichen@redcad.org ." 
"Dr Abdeltif Elbyed obtained his HDR (Ability to Conduct Researches) in Computer Science from the Faculty of Science at Hassan II University of Casablanca. His research interests include E-learning, Interoperability, Ontologies, Semantic web, Multi-agent system and urban transportation. He has published several research papers. He is also Business Intelligence Specialist. His current research interests reverse logistics and urban mobility. Dr Abdeltif Elbyed can be contacted at: a.elbyed@fsac.ac.ma."

"Dr Ismail Assayad obtained his HDR (Ability to Conduct Researches) in Computer Science from ENSEM at Hassan II University of Casablanca. His research interests include Embedded system, Model-Based Conformance/Load/Security Testing Methodologies, SystemC Simulation, Parallelism. Dr Ismail Assayad can be contacted at: iassayad@gmail.com ."

\section{Acknowledgements}

Not applicable.

\section{References}

1. Danai Koutra and Christos Faloutsos. Individual and Collective Graph Mining: Principles, Algorithms, and Applications. Synthesis Lectures on Data Mining and Knowledge Discovery, 9(2), 2017.

2. Byoung-Ha Yoon, Seon-Kyu Kim, and Seon-Young Kim. Use of Graph Database for the Integration of Heterogeneous Biological Data. Genomics \& Informatics, 15(1):19-27, March 2017.

3. Sabeur Aridhi and Engelbert Mephu Nguifo. Big Graph Mining: Frameworks and Techniques. Big Data Research, 6:1-10, 2016.

4. Meng Jiang, Peng Cui, Alex Beutel, Christos Faloutsos, and Shiqiang Yang. Catching Synchronized Behaviors in Large Networks: A Graph Mining Approach. ACM Transactions on Knowledge Discovery from Data, 10(4):1-27, 2016

5. V. E. Alekseev, R. Boliac, D. V. Korobitsyn, and V. V. Lozin. NP-hard graph problems and boundary classes of graphs. Theoretical Computer Science, 389(1):219-236, 2007.

6. Kathie Cameron, Elaine M. Eschen, Chính T. Hoáng, and R. Sritharan. The Complexity of the List Partition Problem for Graphs. SIAM Journal on Discrete Mathematics, 21(4):900-929, 2008.

7. Jonathan Cohen. Graph twiddling in a MapReduce world. Computing in Science E Engineering, 11:29-41, 2009

8. Hamilton Wilfried Yves Adoni, Tarik Nahhal, Moez Krichen, Brahim Aghezzaf, and Abdeltif Elbyed. A survey of current challenges in partitioning and processing of graph-structured data in parallel and distributed systems. Distributed and Parallel Databases, 38(2):495-530, 2020.

9. Sabeur Aridhi, Philippe Lacomme, Libo Ren, and Benjamin Vincent. A mapreduce-based approach for shortest path problem in large-scale networks. Engineering Applications of Artificial Intelligence, 41:151-165, 2015 .

10. Boris V. Cherkassky, Andrew V. Goldberg, and Tomas Radzik. Shortest paths algorithms: Theory and experimental evaluation. Mathematical Programming, 73:129-174, 1993.

11. Wilfried Yves Hamilton Adoni, Tarik Nahhal, Brahim Aghezzaf, and Abdeltif Elbyed. MRA*: Parallel and Distributed Path in Large-Scale Graph Using MapReduce-A* Based Approach. In Ubiquitous Networking, Lecture Notes in Computer Science, pages 390-401. Springer, Cham, May 2017.

12. Wilfried Yves Hamilton Adoni, Tarik Nahhal, Brahim Aghezzaf, and Abdeltif Elbyed. The MapReduce-based approach to improve the shortest path computation in large-scale road networks: the case of $\mathrm{A}^{*}$ algorithm. Journal of Big Data, 5, 2018.

13. Sabeur Aridhi, Alberto Montresor, and Yannis Velegrakis. BLADYG: A Graph Processing Framework for Large Dynamic Graphs. Big Data Research, 9:9-17, 2017.

14. Vinod Kumar Vavilapalli, Siddharth Seth, Bikas Saha, Carlo Curino, Owen O'Malley, Sanjay Radia, Benjamin Reed, Eric Baldeschwieler, Arun C. Murthy, Chris Douglas, Sharad Agarwal, Mahadev Konar, Robert Evans, Thomas Graves, Jason Lowe, and Hitesh Shah. Apache hadoop YARN: yet another resource negotiator. In Proceedings of the 4th Annual Symposium on Cloud Computing, pages 1-16, Santa Clara, California, 2013. ACM Press. 
15. Matei Zaharia, Mosharaf Chowdhury, Michael J. Franklin, Scott Shenker, and Ion Stoica. Spark: Cluster computing with working sets. HotCloud, 10(10-10):95, 2010.

16. Badr Ait Hammou, Ayoub Ait Lahcen, and Salma Mouline. APRA: An approximate parallel recommendation algorithm for Big Data. Knowledge-Based Systems, 157:10-19, 2018.

17. Eric Brewer. Pushing the CAP: Strategies for Consistency and Availability. Computer, 45(2):23-29, 2012.

18. Ana Paula Appel and Luis G. Moyano. Link and Graph Mining in the Big Data Era. In Albert Y. Zomaya and Sherif Sakr, editors, Handbook of Big Data Technologies, pages 583-616. Springer International Publishing, Cham, 2017.

19. Jon Louis Bentley. Multidimensional Divide-and-conquer. Commun. ACM, 23(4):214-229, 1980.

20. Kijung Shin, Tina Eliassi-Rad, and Christos Faloutsos. CoreScope: Graph Mining Using k-Core Analysis Patterns, Anomalies and Algorithms. In 2016 IEEE 16th International Conference on Data Mining (ICDM), pages 469-478, December 2016. ISSN: 2374-8486.

21. Alessio Guerrieri. Distributed computing for large-scale graphs. PhD thesis, University of Trento, 2015.

22. Fatemeh Rahimian, Amir H. Payberah, Sarunas Girdzijauskas, Mark Jelasity, and Seif Haridi. A Distributed Algorithm for Large-Scale Graph Partitioning. ACM Transactions on Autonomous and Adaptive Systems, 10(2):1-24, 2015.

23. Joseph E. Gonzalez, Yucheng Low, Haijie Gu, Danny Bickson, and Carlos Guestrin. PowerGraph: Distributed Graph-parallel Computation on Natural Graphs. In Proceedings of the 10th USENIX Conference on Operating Systems Design and Implementation, OSDI'12, pages 17-30, Berkeley, CA, USA, 2012. USENIX Association.

24. Fatemeh Rahimian, Amir H. Payberah, Sarunas Girdzijauskas, and Seif Haridi. Distributed vertex-cut partitioning. In IFIP International Conference on Distributed Applications and Interoperable Systems, pages 186-200. Springer, 2014.

25. Donghui Yan, Ling Huang, and Michael I. Jordan. Fast Approximate Spectral Clustering. In Proceedings of the 15th ACM SIGKDD International Conference on Knowledge Discovery and Data Mining, KDD '09, pages 907-916, New York, NY, USA, 2009. ACM.

26. Charles H. Martin and PhD. Spectral Clustering: A quick overview, 2012.

27. B. W. Kernighan and S. Lin. An efficient heuristic procedure for partitioning graphs. The Bell System Technical Journal, 49(2):291-307, 1970.

28. C. M. Fiduccia and R. M. Mattheyses. A Linear-time Heuristic for Improving Network Partitions. In Proceedings of the 19th Design Automation Conference, DAC '82, pages 175-181, Piscataway, NJ, USA, 1982. IEEE Press.

29. G. Karypis and V. Kumar. A Fast and High Quality Multilevel Scheme for Partitioning Irregular Graphs. SIAM Journal on Scientific Computing, 20:359-392, 1998.

30. George Karypis and Vipin Kumar. Multilevel Algorithms for Multi-constraint Graph Partitioning. In Proceedings of the 1998 ACM/IEEE Conference on Supercomputing, SC '98, pages 1-13, Washington, DC, USA, 1998. IEEE Computer Society.

31. George Karypis and Vipin Kumar. Multilevel K-way Hypergraph Partitioning. In Proceedings of the 36th Annual ACM/IEEE Design Automation Conference, DAC '99, pages 343-348, New York, NY, USA, 1999. ACM.

32. Kirk Schloegel, George Karypis, and Vipin Kumar. Parallel Multilevel Algorithms for Multi-constraint Graph Partitioning. In Euro-Par 2000 Parallel Processing, Lecture Notes in Computer Science, pages 296-310. Springer, Berlin, Heidelberg, 2000.

33. Aapo Kyrola, Guy Blelloch, and Carlos Guestrin. GraphChi: Large-scale Graph Computation on Just a PC. In Proceedings of the 10th USENIX Conference on Operating Systems Design and Implementation, OSDI'12, pages 31-46, Berkeley, CA, USA, 2012. USENIX Association.

34. Charalampos Tsourakakis, Christos Gkantsidis, Bozidar Radunovic, and Milan Vojnovic. FENNEL: Streaming Graph Partitioning for Massive Scale Graphs. In Proceedings of the 7th ACM International Conference on Web Search and Data Mining, WSDM '14, pages 333-342, New York, NY, USA, 2014. ACM.

35. Charu C. Aggarwal, Yuchen Zhao, and Philip S. Yu. A framework for clustering massive graph streams. Statistical Analysis and Data Mining, 3(6):399-416, 2010.

36. Edward Kao, Vijay Gadepally, Michael Hurley, Michael Jones, Jeremy Kepner, Sanjeev Mohindra, Paul Monticciolo, Albert Reuther, Siddharth Samsi, William Song, Diane Staheli, and Steven Smith. Streaming graph challenge: Stochastic block partition. In 2017 IEEE High Performance Extreme Computing Conference (HPEC), pages 1-12, 2017.

37. Isabelle Stanton and Gabriel Kliot. Streaming Graph Partitioning for Large Distributed Graphs. In Proceedings of the 18th ACM SIGKDD International Conference on Knowledge Discovery and Data Mining, KDD '12, pages 1222-1230, New York, NY, USA, 2012. ACM.

38. Katerina Tashkova, P. Korošec, and J. Silc. A distributed multilevel ant-colony algorithm for the multi-way graph partitioning. International Journal of Bio-Inspired Computation, 3(5):286-296, 2011.

39. Hayato Ushijima-Mwesigwa, Christian F. A. Negre, and Susan M. Mniszewski. Graph Partitioning using Quantum Annealing on the D-Wave System. In Proceedings of the Second International Workshop on Post Moores Era Supercomputing, PMES'17, pages 22-29, Denver, CO, USA, 2017. Association for Computing Machinery. 
40. Henning Meyerhenke, Peter Sanders, and Christian Schulz. Parallel Graph Partitioning for Complex Networks. IEEE Transactions on Parallel and Distributed Systems, 28(9):2625-2638, 2017.

41. Leslie G. Valiant. A Bridging Model for Parallel Computation. Commun. ACM, 33(8):103-111, 1990.

42. Matthew L Massie, Brent N Chun, and David E Culler. The ganglia distributed monitoring system: design, implementation, and experience. Parallel Computing, 30(7):817-840, 2004.

43. Martin Junghanns, Andre Petermann, Niklas Teichmann, Kevin Gomez, and Erhard Rahm. Analyzing extended property graphs with Apache Flink. In Proceedings of the 1st ACM SIGMOD Workshop on Network Data Analytics - NDA '16, pages 1-8, San Francisco, California, 2016. ACM Press.

44. Joseph E. Gonzalez, Reynold S. Xin, Ankur Dave, Daniel Crankshaw, Michael J. Franklin, and Ion Stoica. Graphx: Graph processing in a distributed dataflow framework. In 11th $\$\{\$$ USENIX\$\}\$ Symposium on Operating Systems Design and Implementation (\$\{\$SDI\$\}\$14), pages 599-613, 2014.

45. Konstantin Shvachko, Hairong Kuang, Sanjay Radia, and Robert Chansler. The Hadoop Distributed File System. In Proceedings of the 2010 IEEE 26th Symposium on Mass Storage Systems and Technologies (MSST), pages 1-10. IEEE Computer Society, 2010. 
Figures

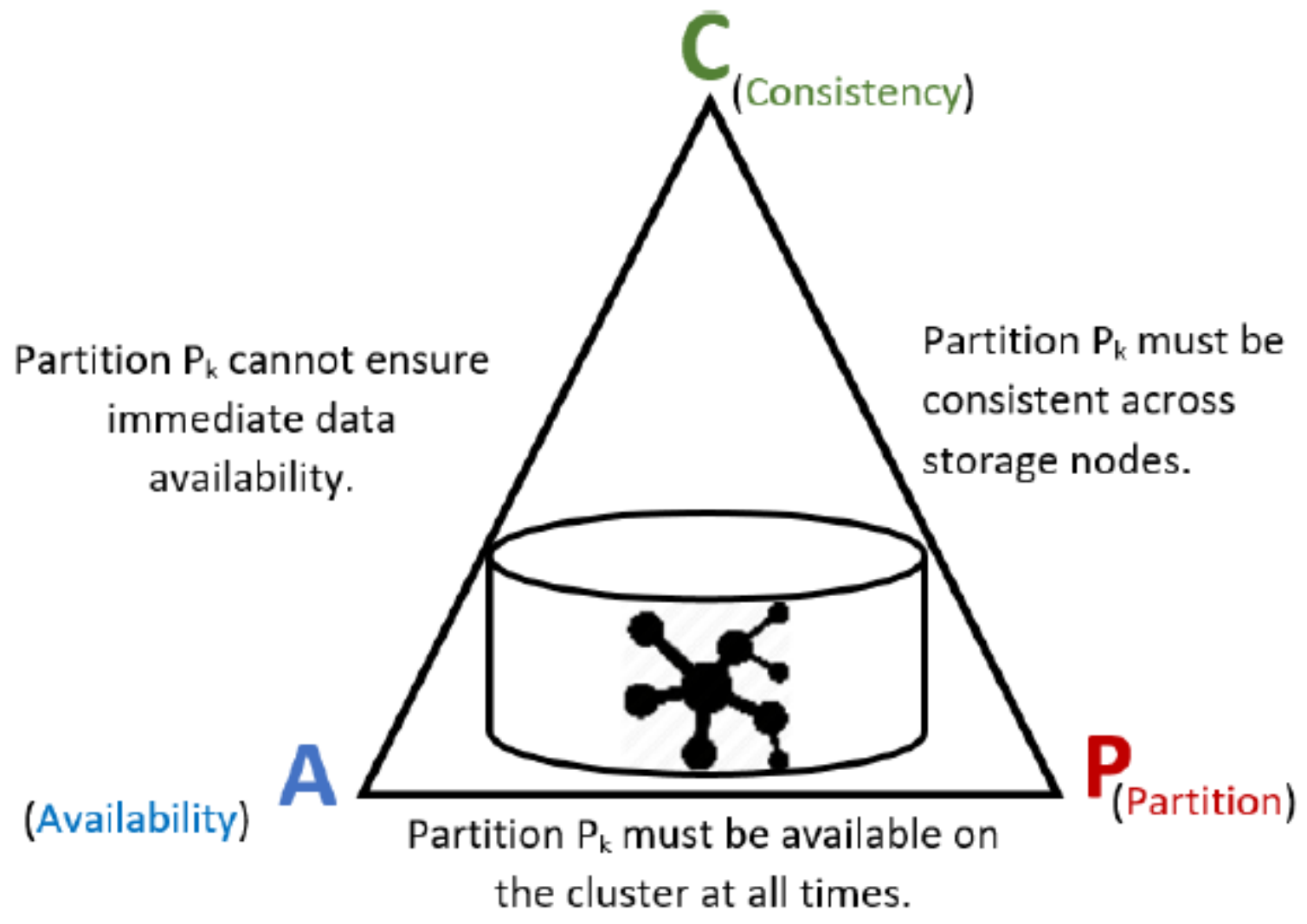

Figure 1

CAP theorem 


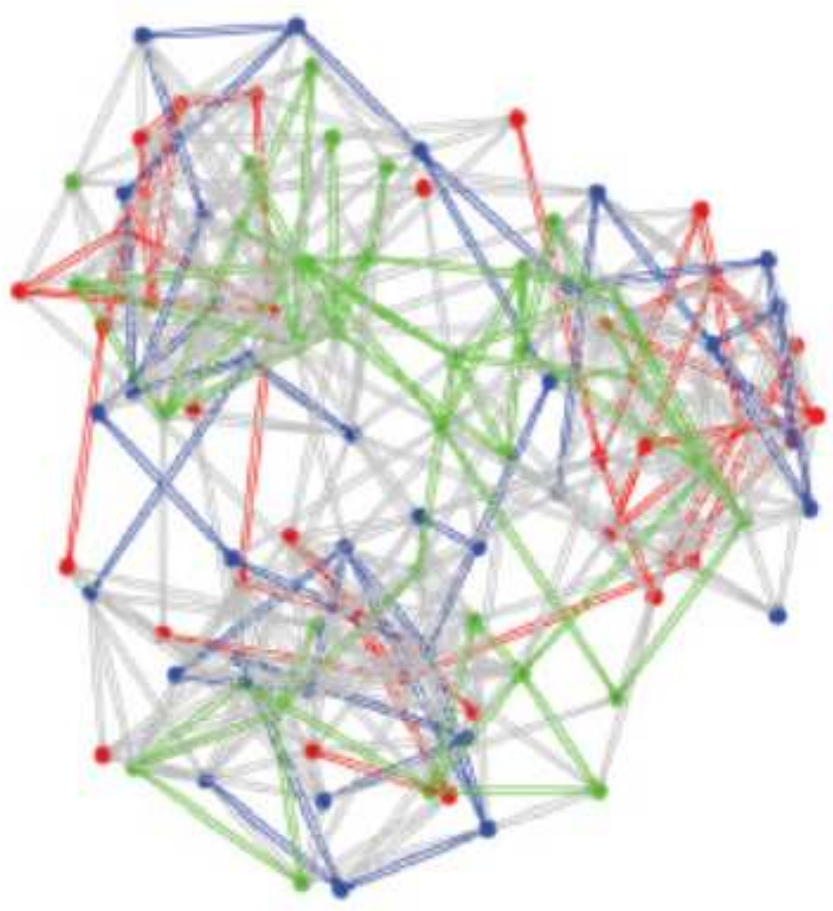

(a)

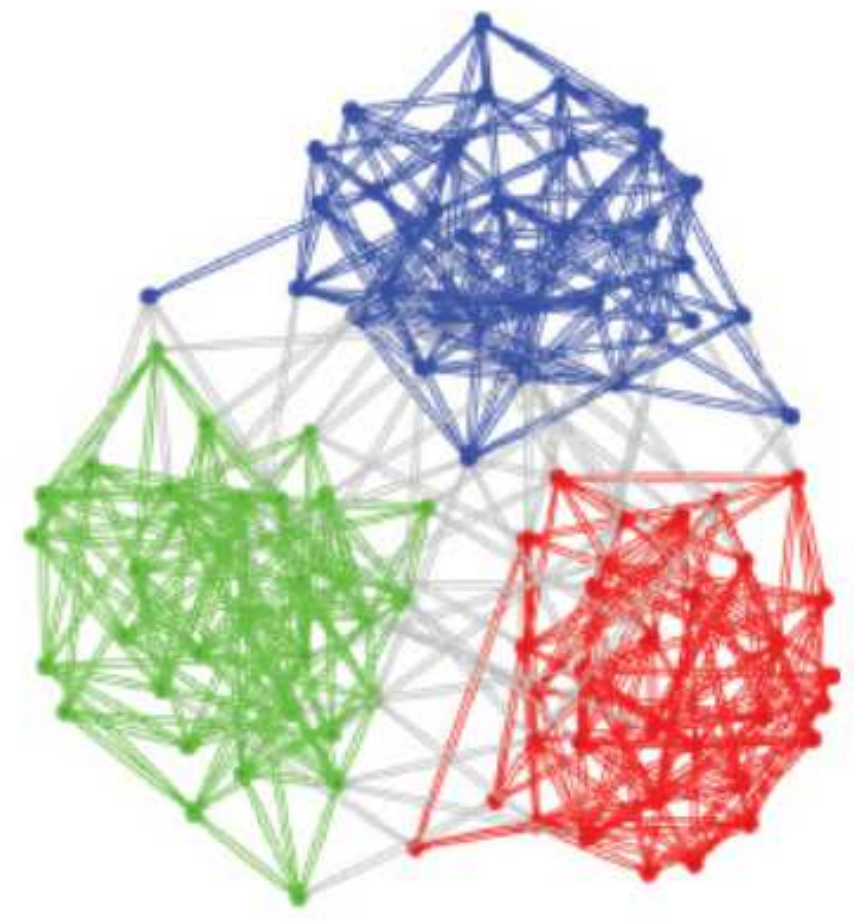

(b)

Figure 2

Illustration of an example of partitioning : (a) the partitioning is shoddy, the vertices are assigned randomly, there are more cut-edges between partitions; (b) the partitioning is almost optimal, the highly connected vertices reside in the same partition, there is less cut-edges [25]. 


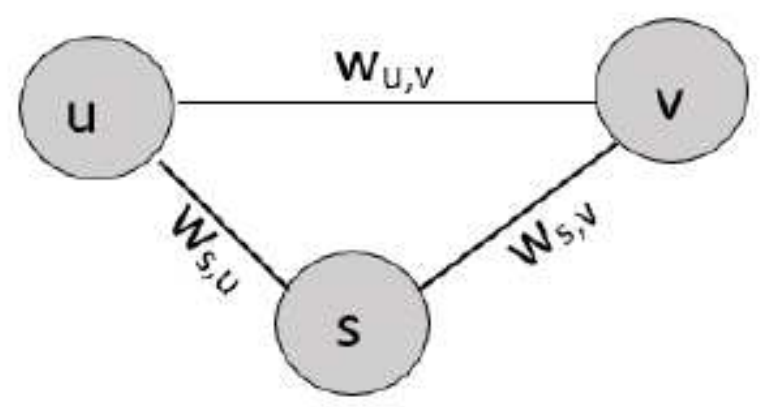

(a)

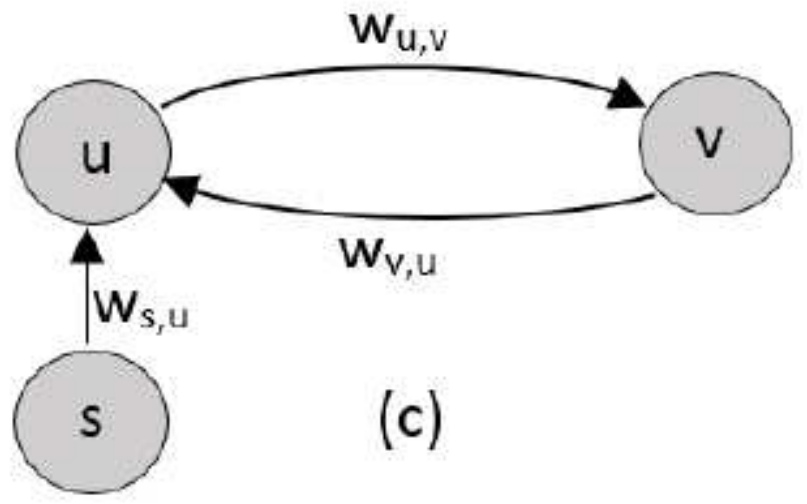

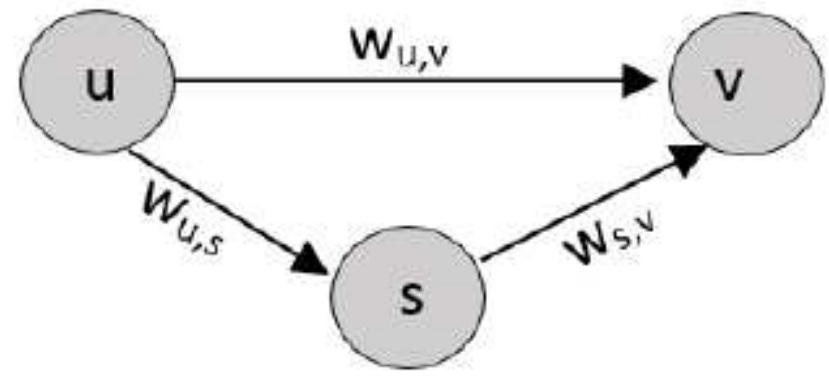

(b)

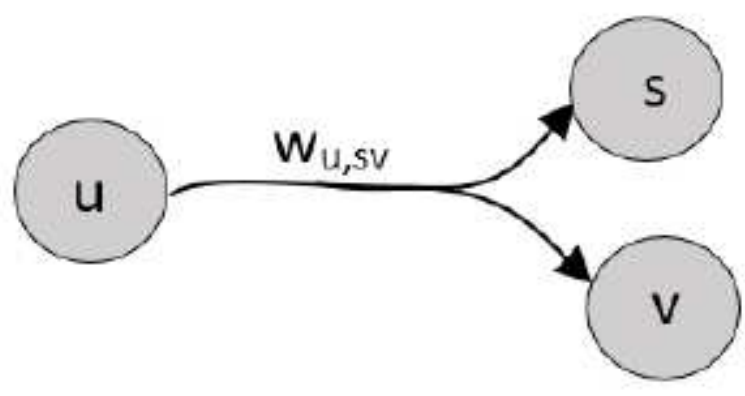

(d)

Figure 3

Various types of graphs: (a) undirected graph; (b) directed graph; (c) multi-graph; (d) hyper-graph.

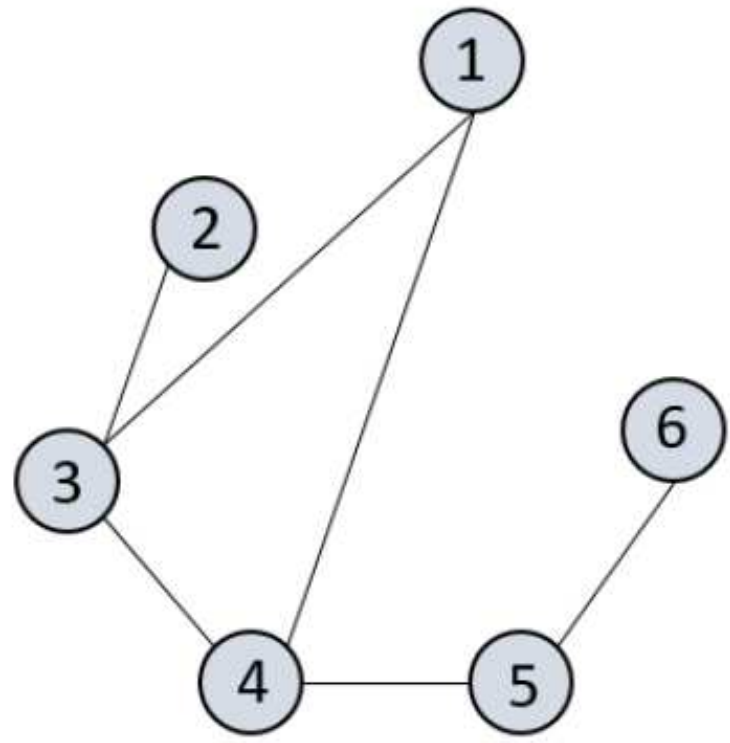

(a)

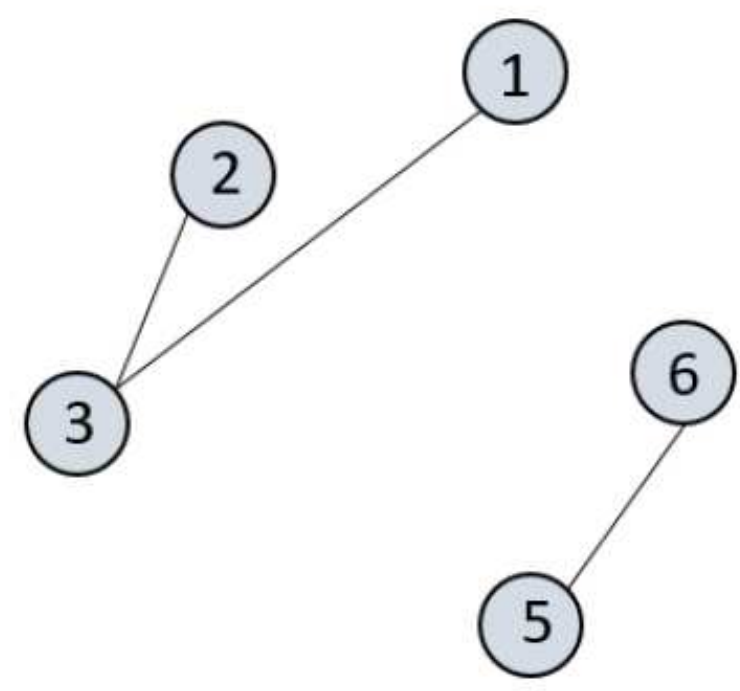

(b)

(a) graph $G r=(V r, E d)$; (b) sub-graph $G r^{\prime}=\left(V r^{\prime}, E d^{\prime}\right)$. 
(a) graph $G r=(V r, E d) ;(b)$ sub-graph $G r^{\prime}=\left(V r^{\prime}, E d '\right)$.

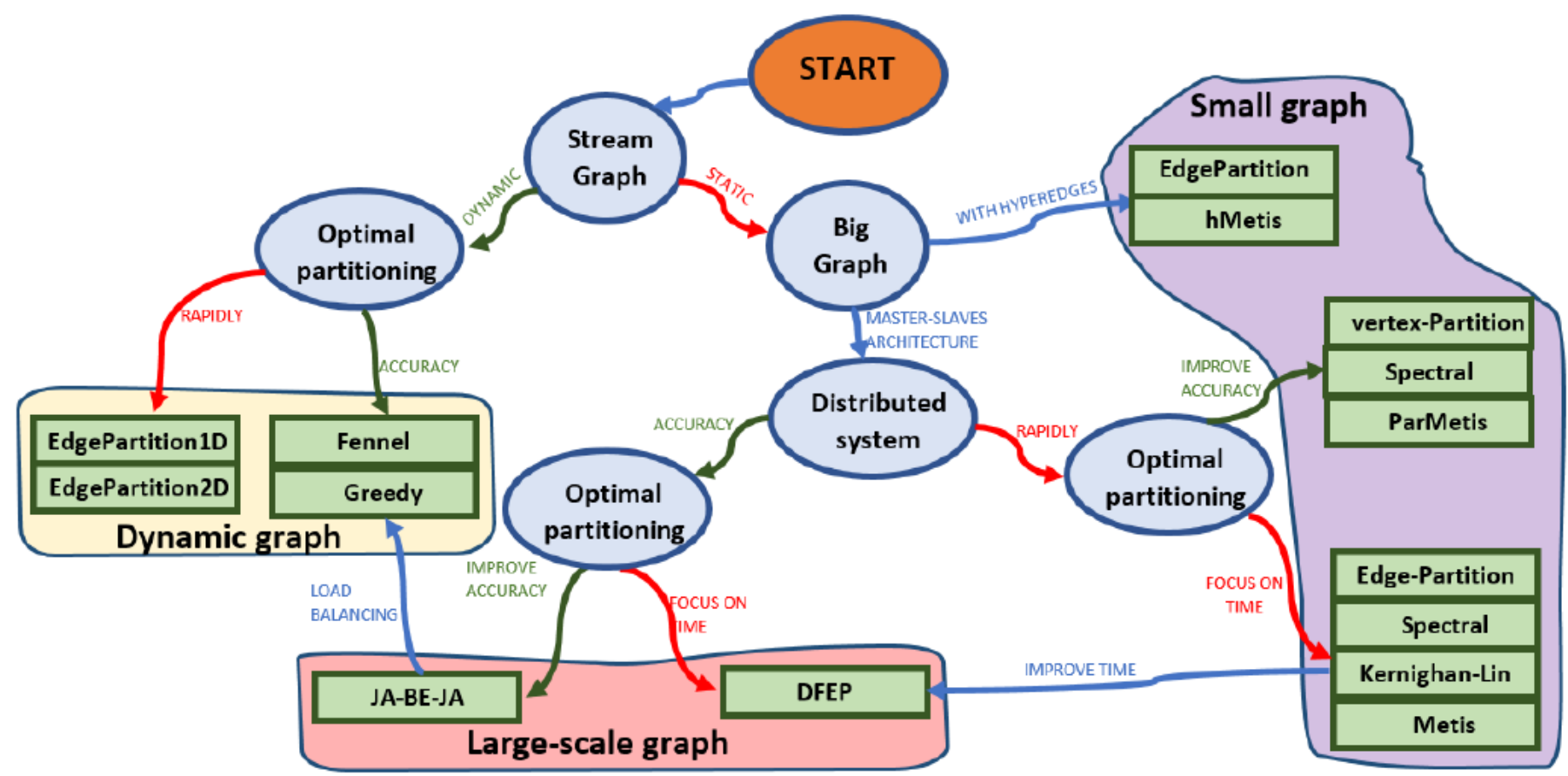

Figure 5

Roadmap of graph partitioning algorithms

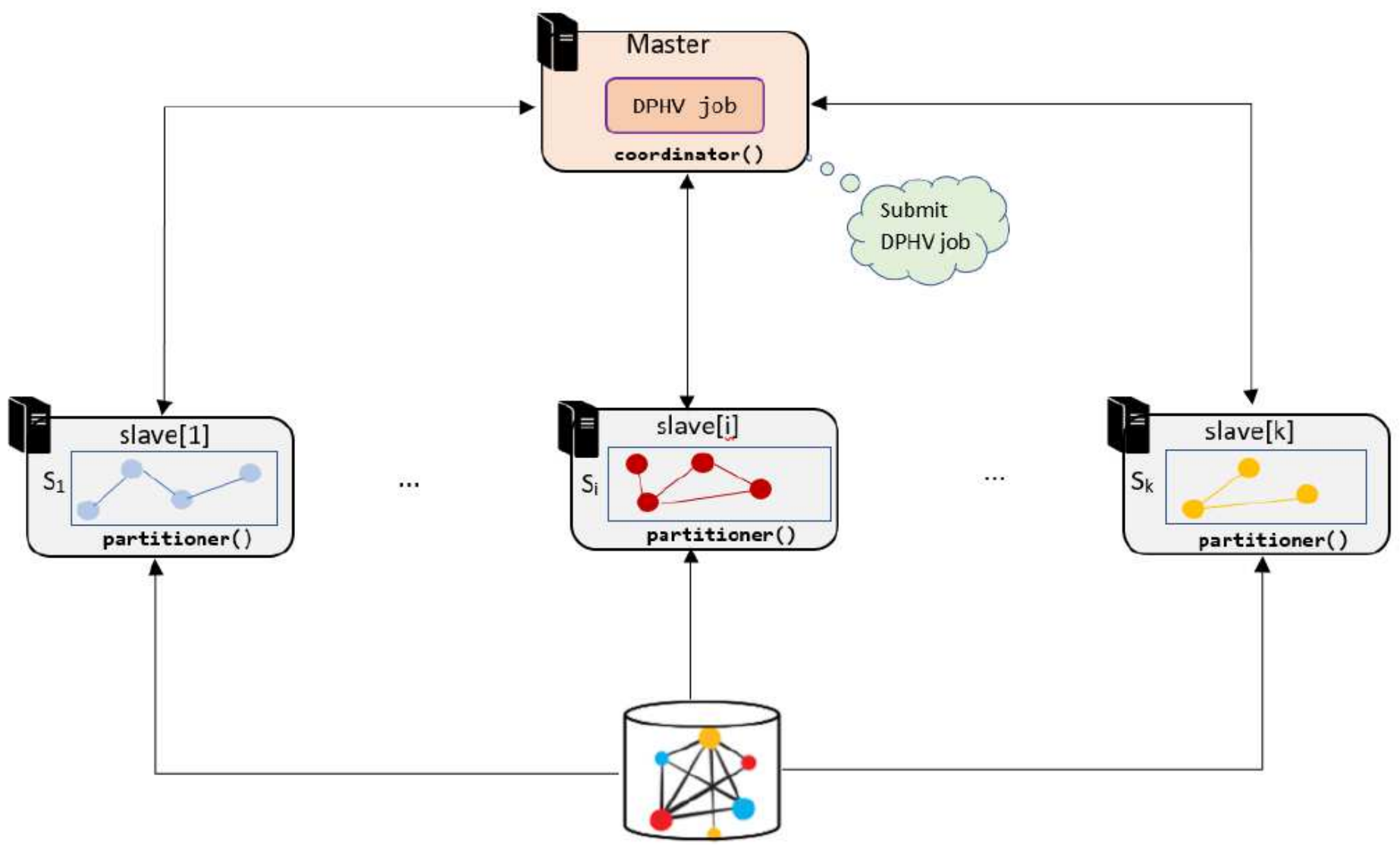

Figure 6 


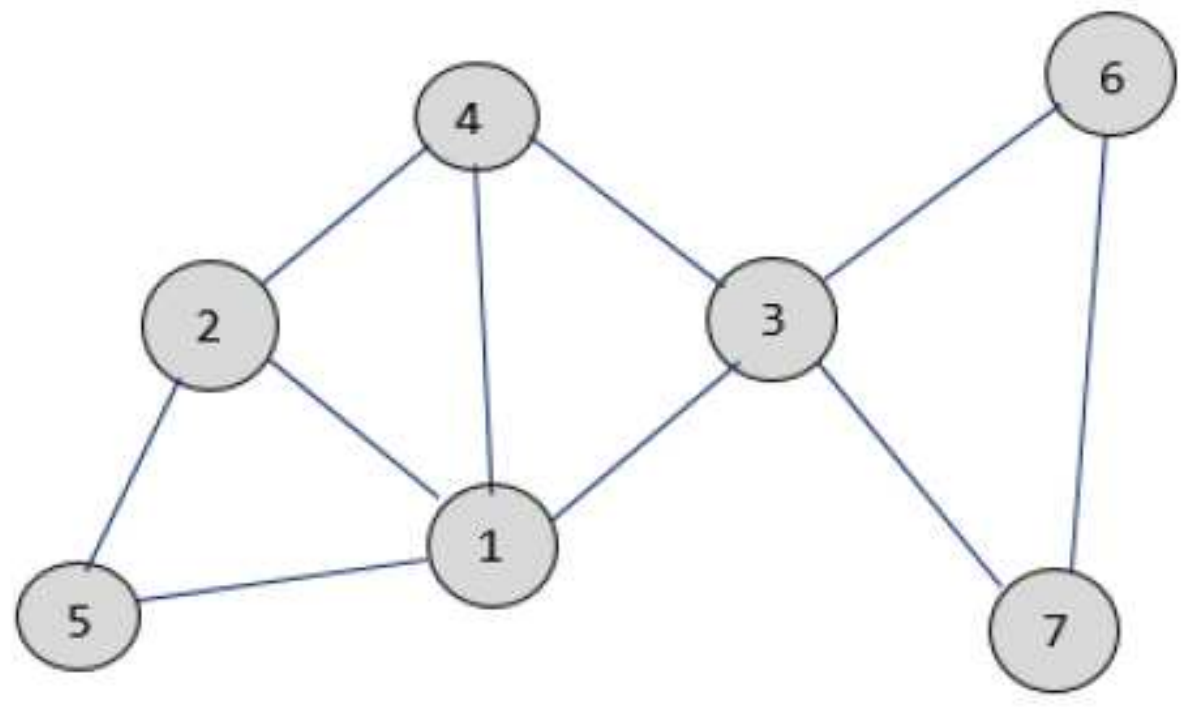

Figure 7

Graph GR $=(\mathrm{Vr}, \mathrm{Ed})$ such as $|\mathrm{Vr}|=7 \mathrm{et}|\mathrm{Ed}|=10$
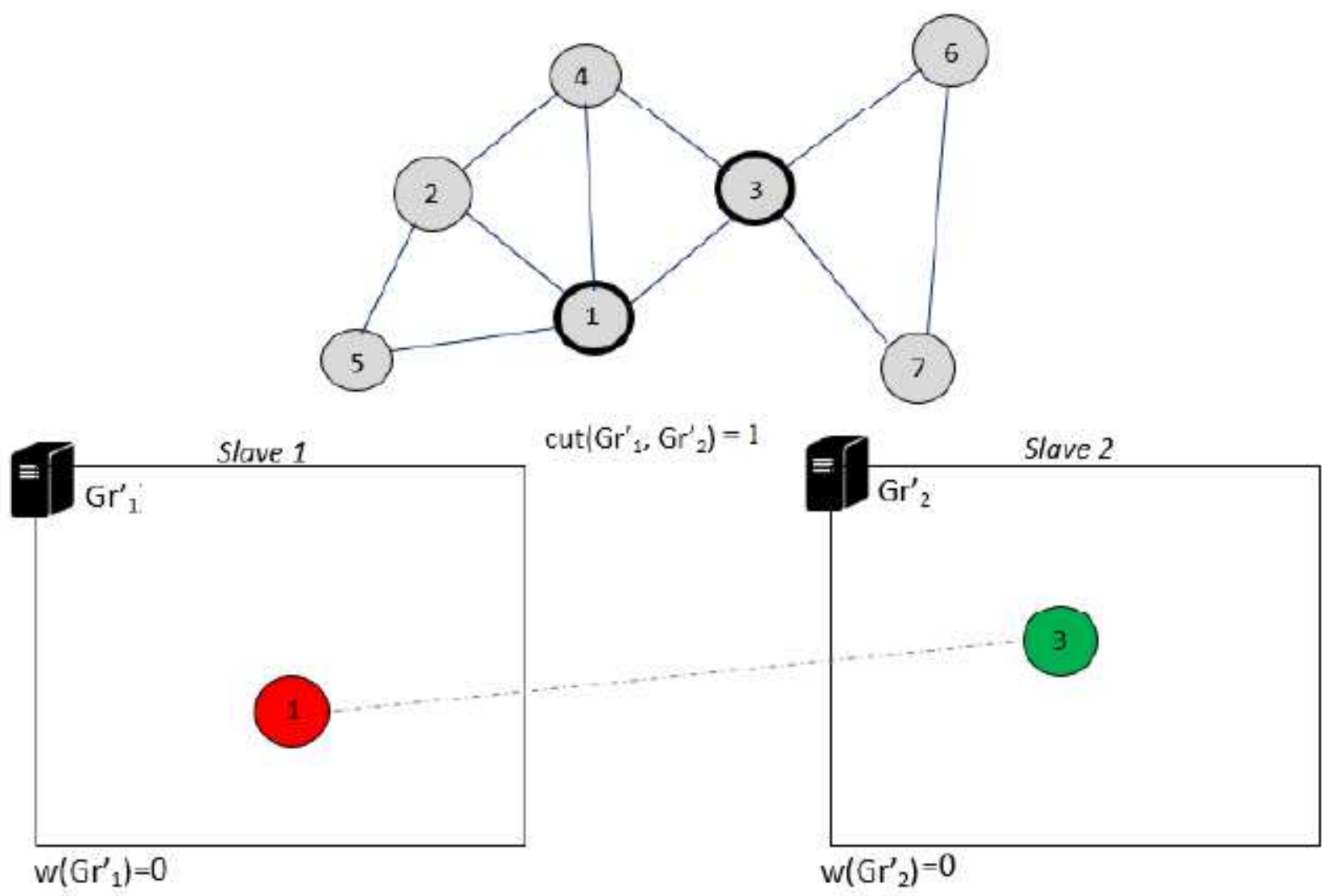

Figure 8 
Iteration 1 of DPHV execution
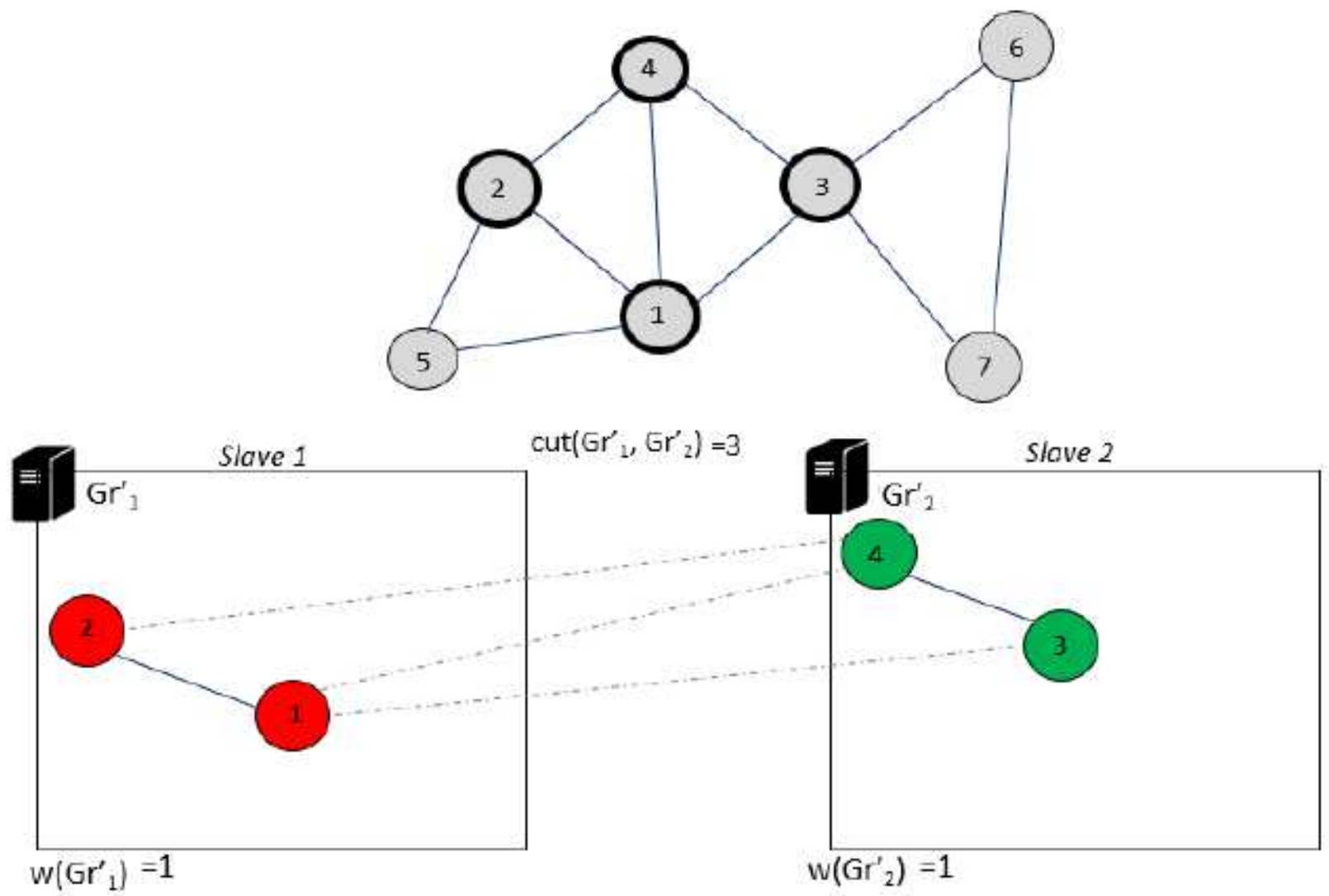

Figure 9

Iteration 2 of DPHV execution 

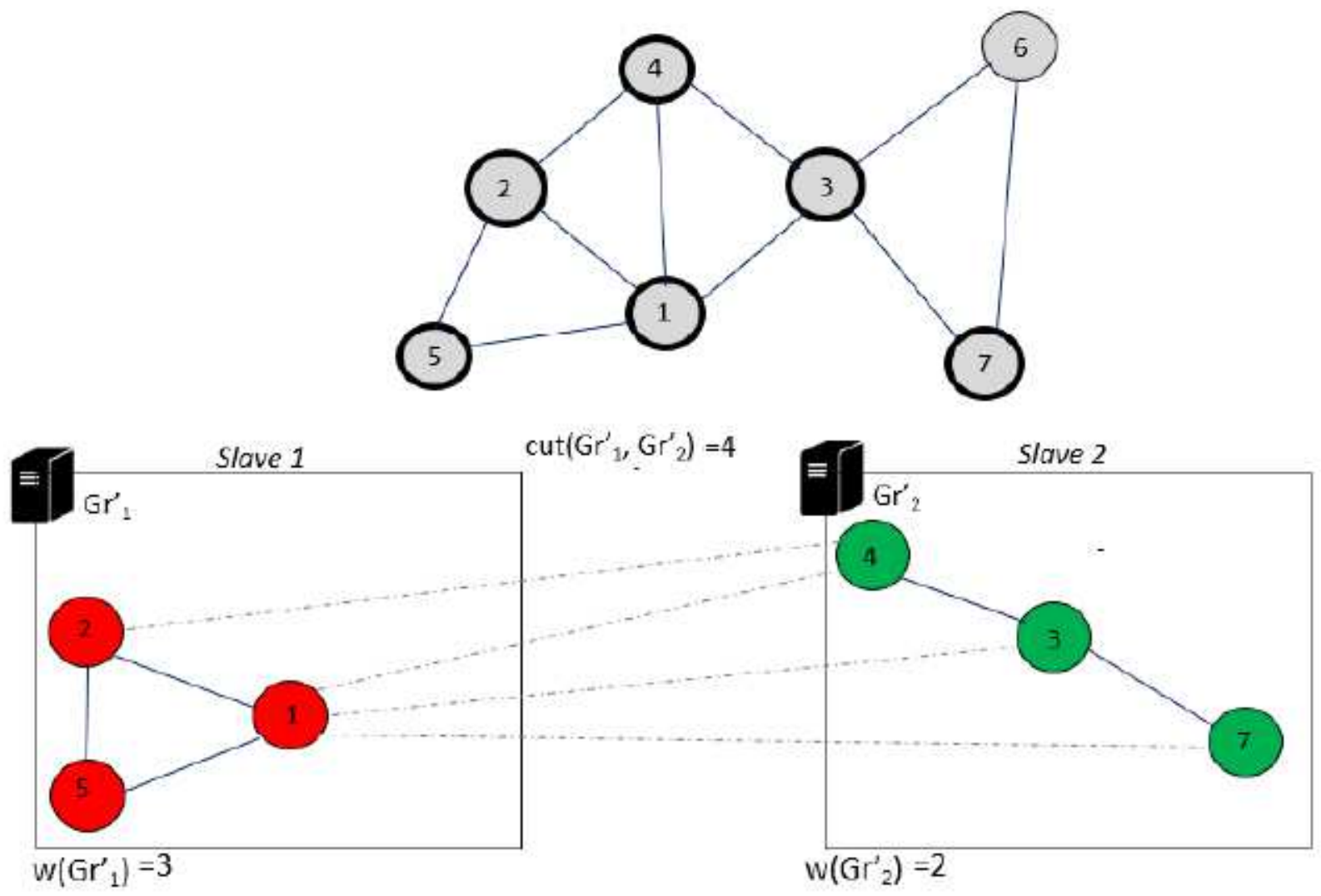

Figure 10

Iteration 3 of DPHV execution 

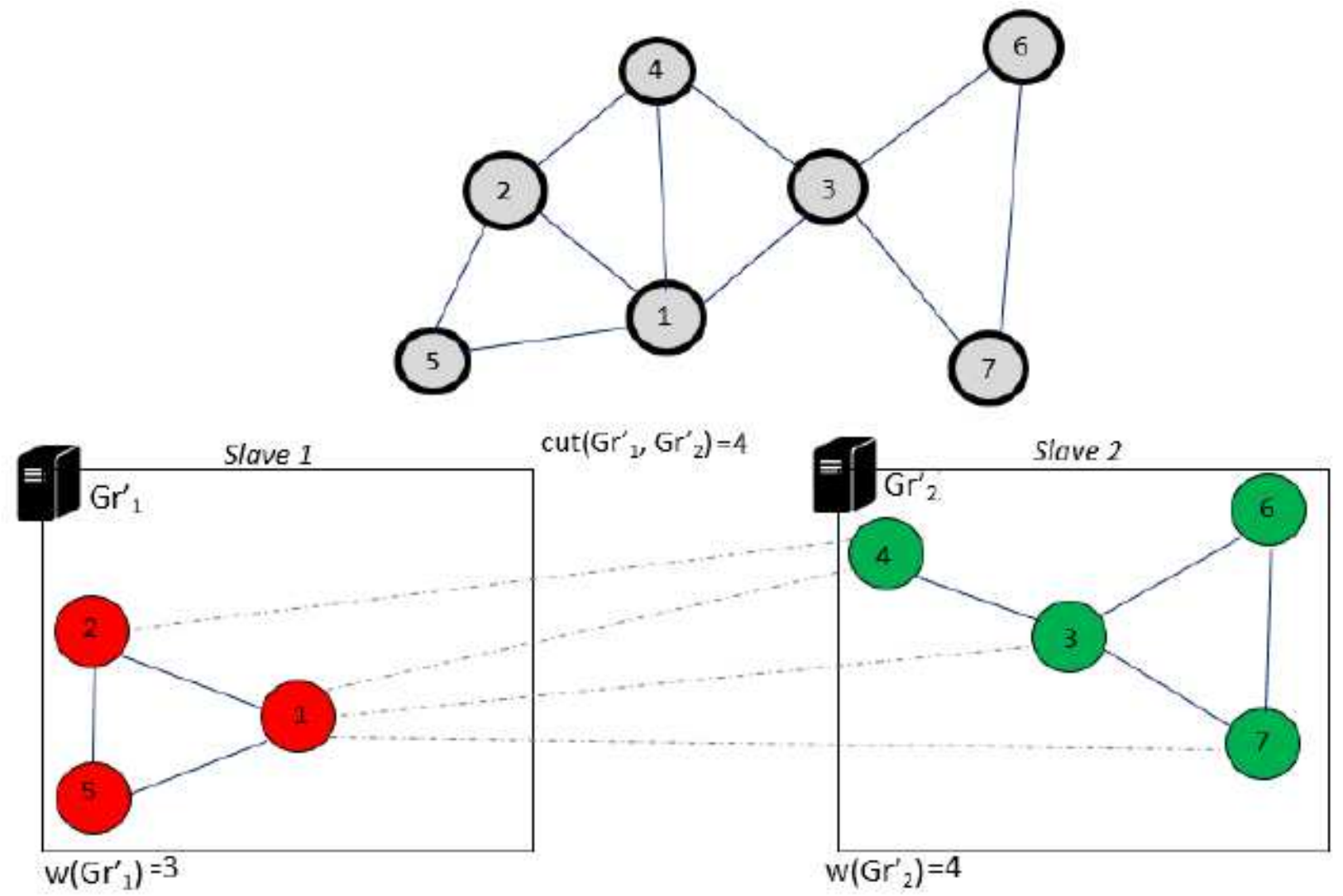

Figure 11

Iteration 4 of DPHV execution 


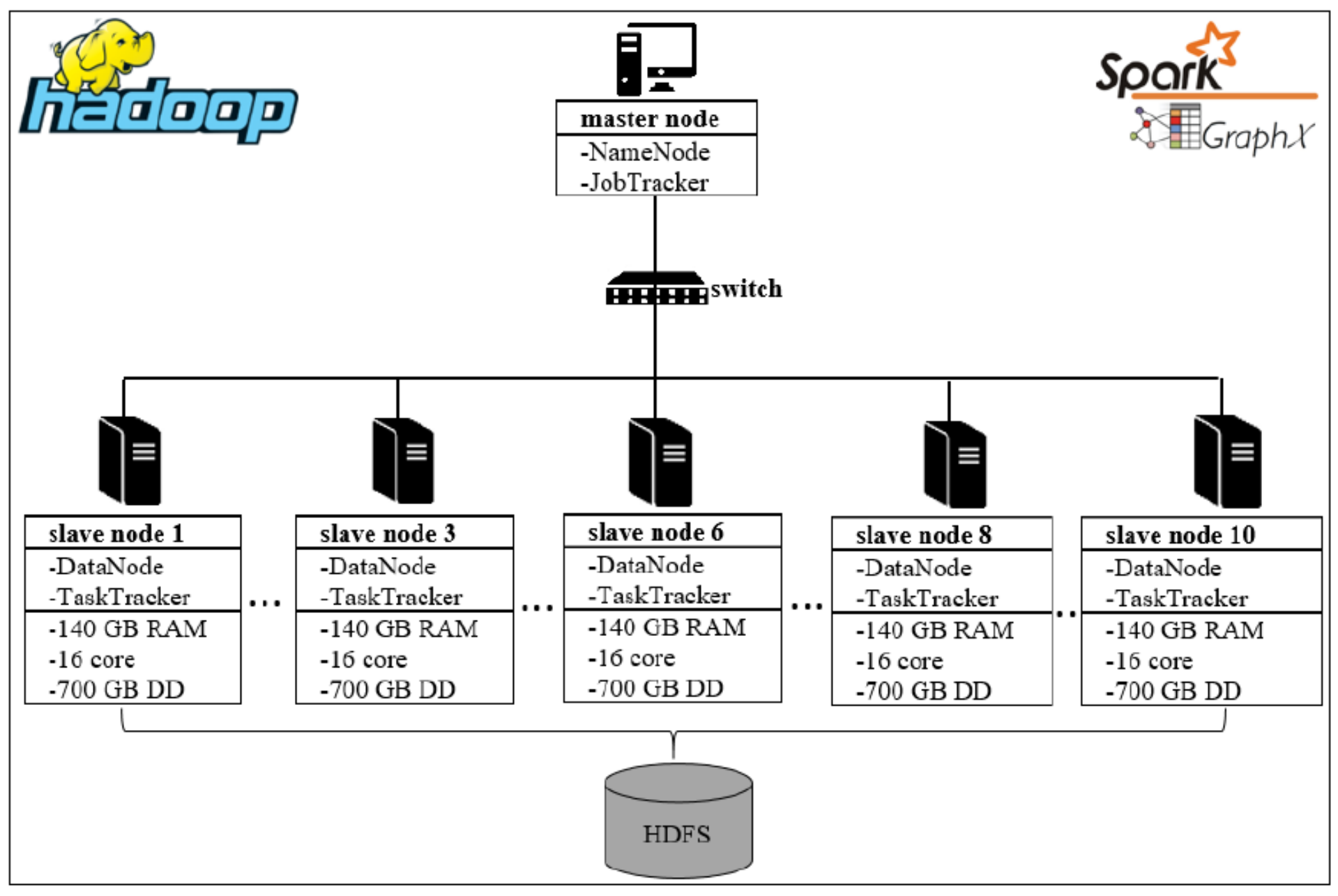

Figure 12

Test environment 


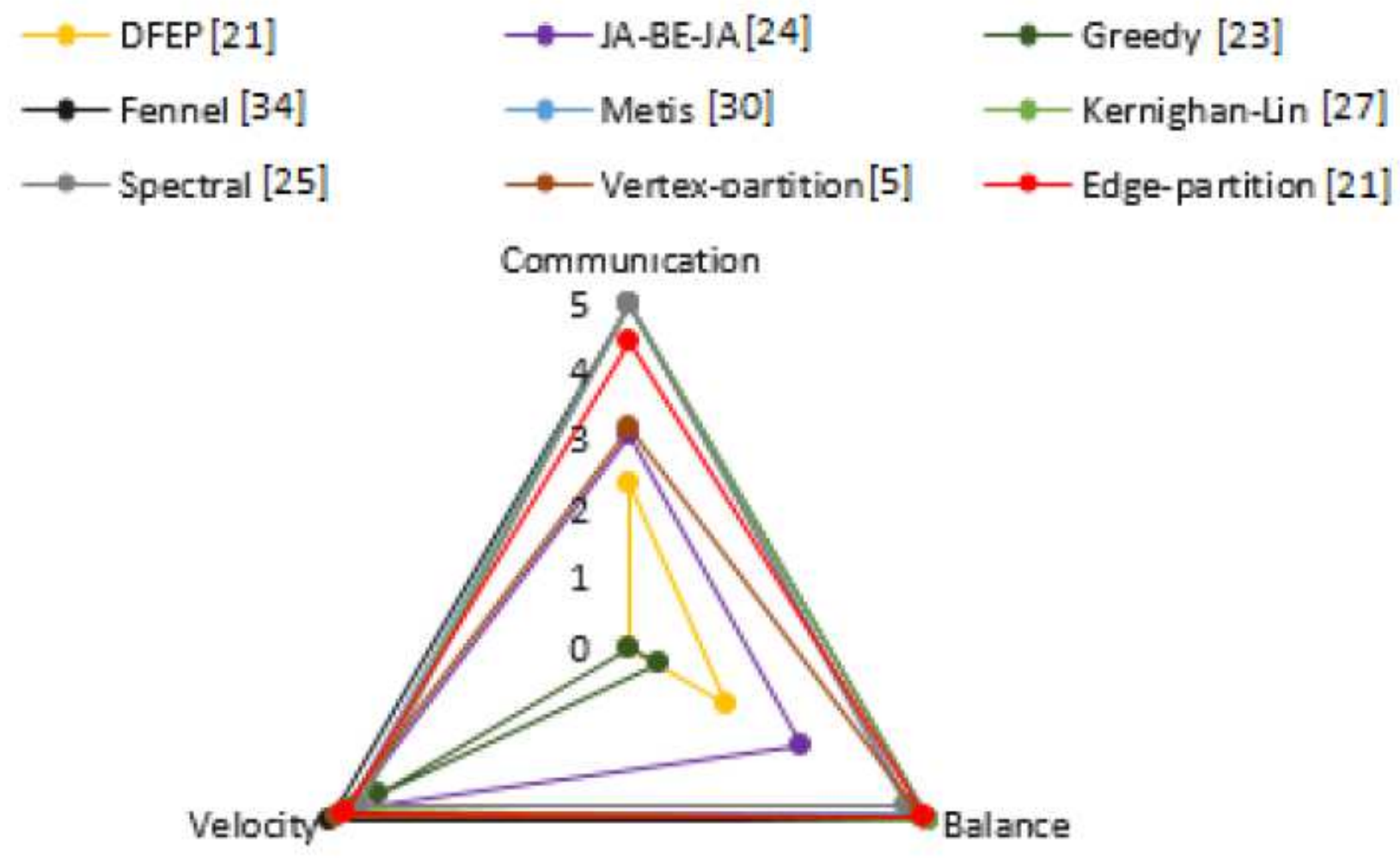

Figure 13

Computational complexity of graph partitioning methods 

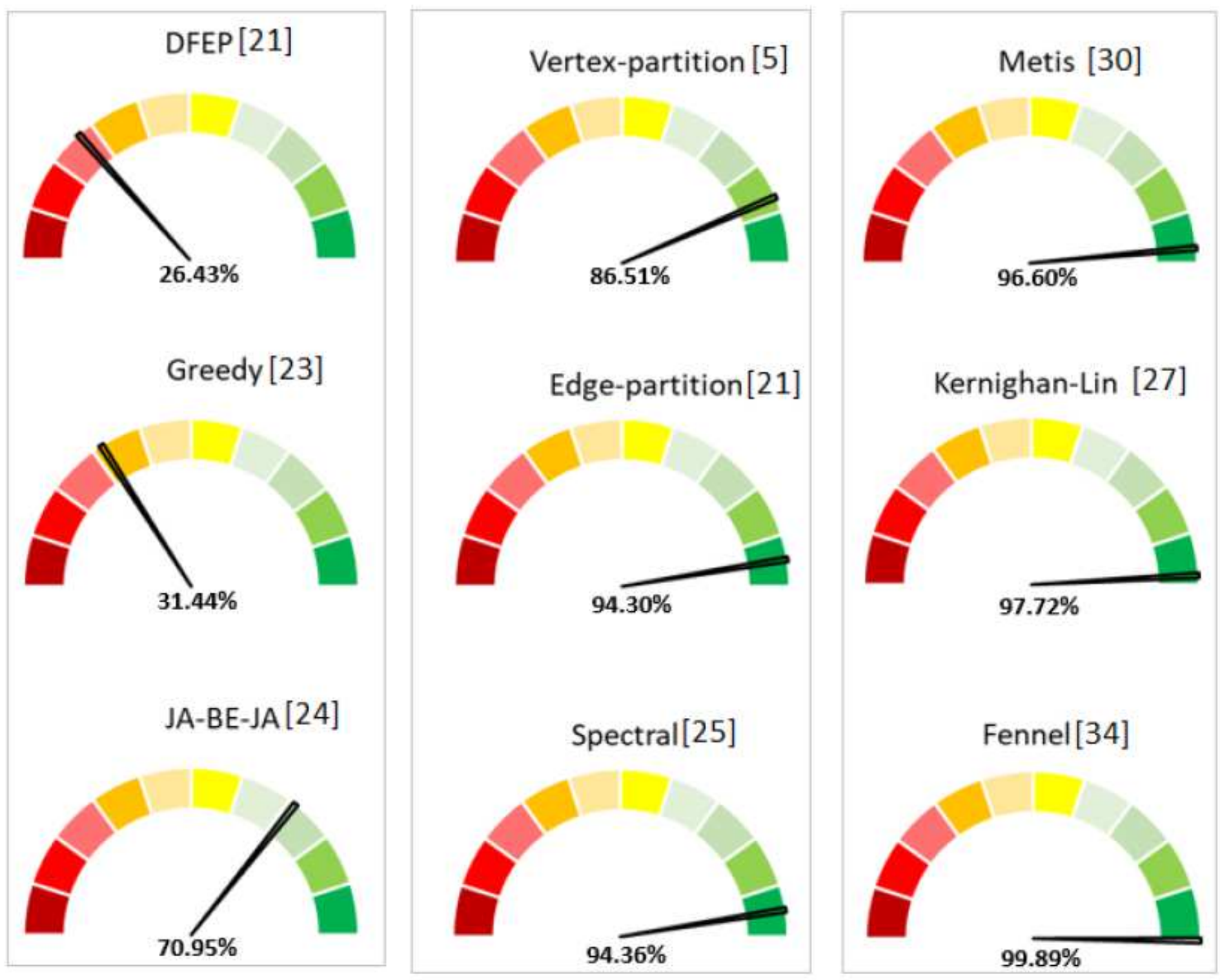

Figure 14

Constraints satisfaction rate 
(a) Runtime

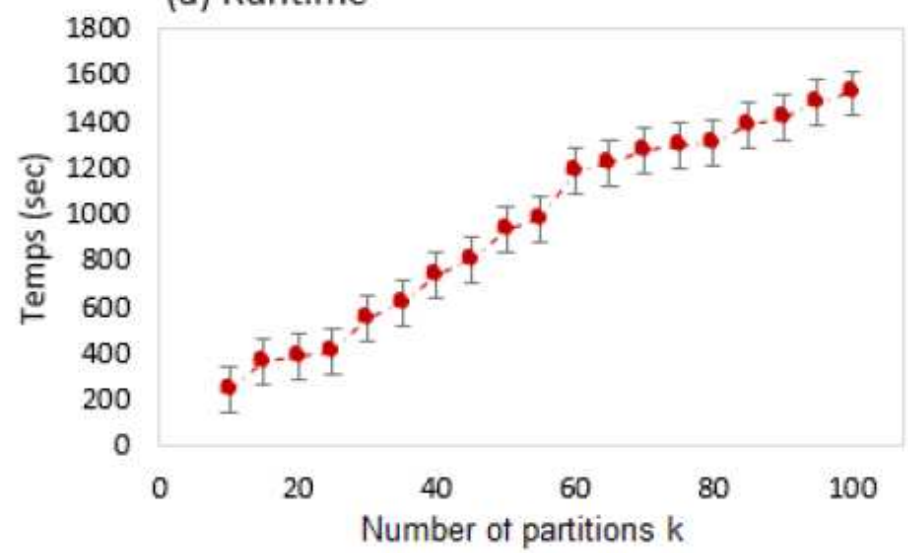

(c) Balance

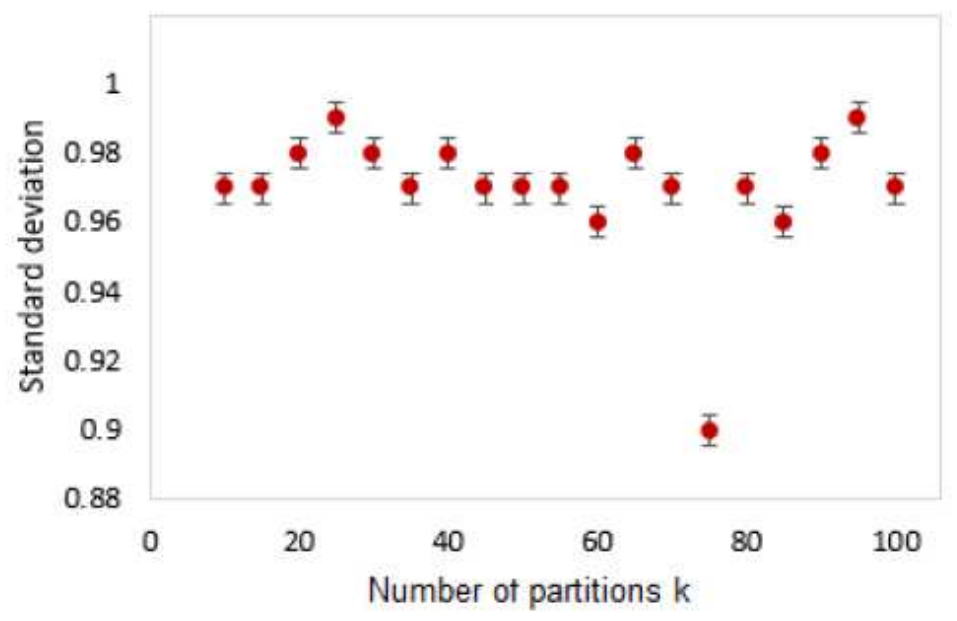

(b) Cross-edges between partitions

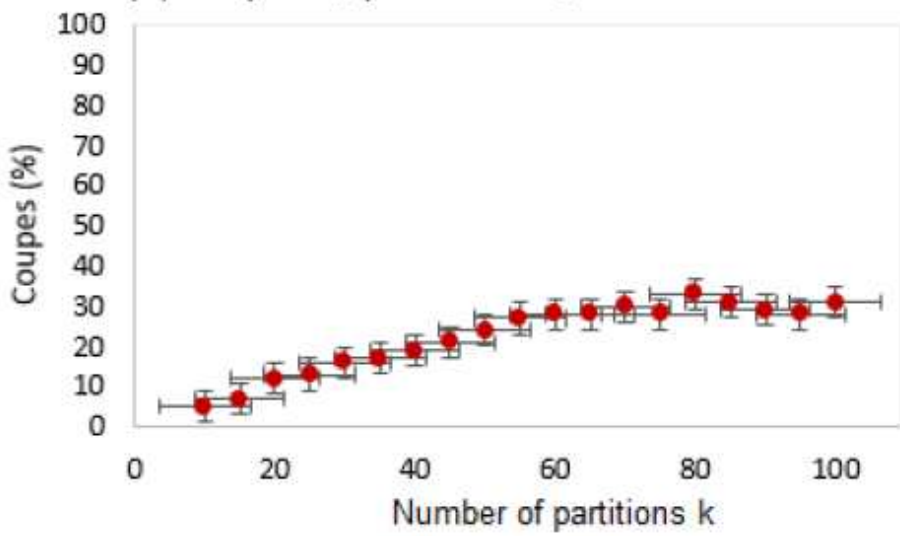

(d) Connectivity of induced sub-graphs

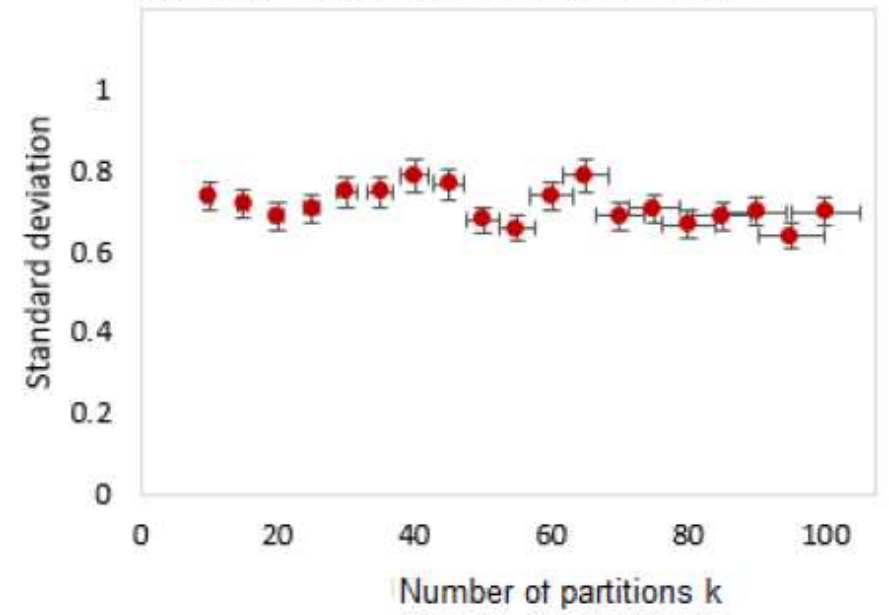

Figure 15

DPHV behavior based on the number of partition $\mathrm{k}$ 
(a) Runtime

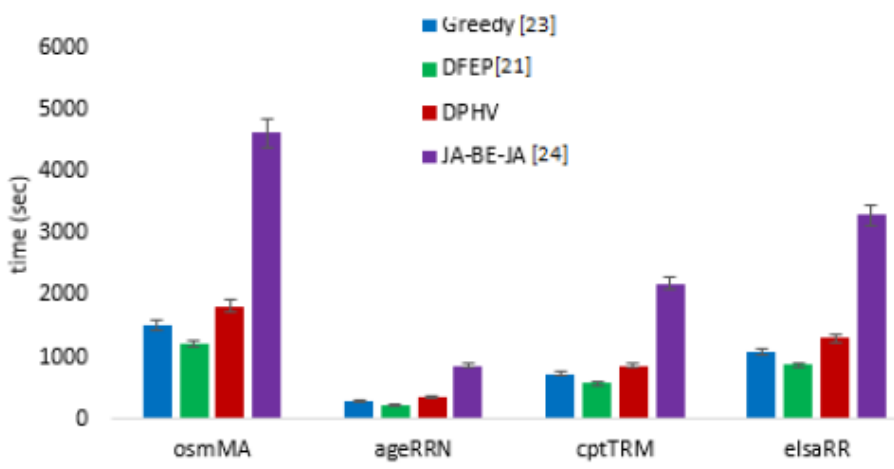

(c) Balance

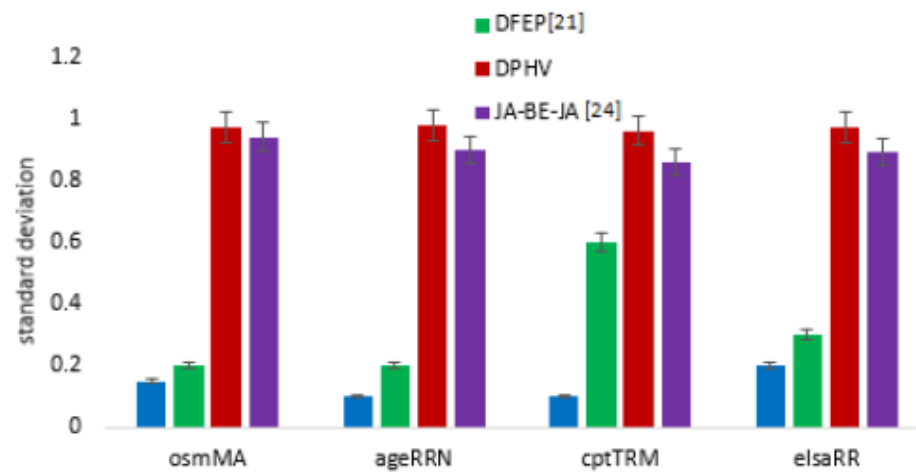

(b) Croșs-edges between partitions

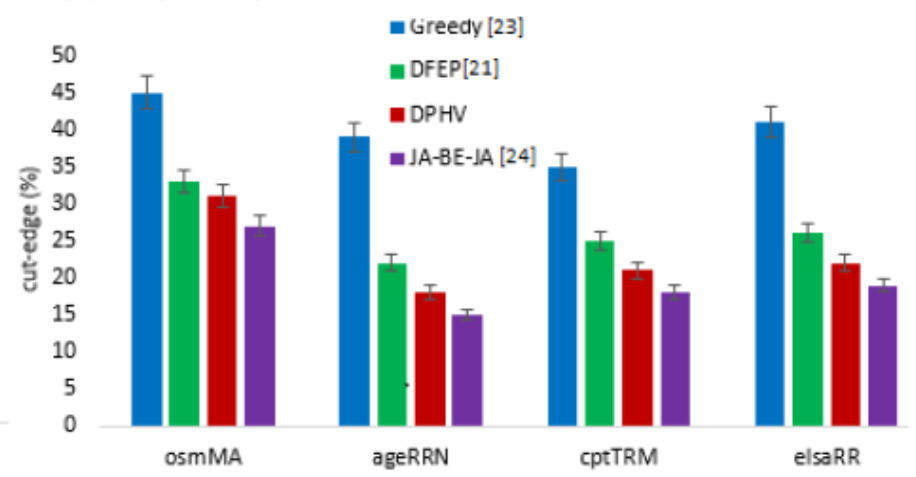

(d) Connectivity of induced sub-graphs

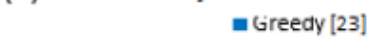

$$
\text { - Greedy [23] }
$$

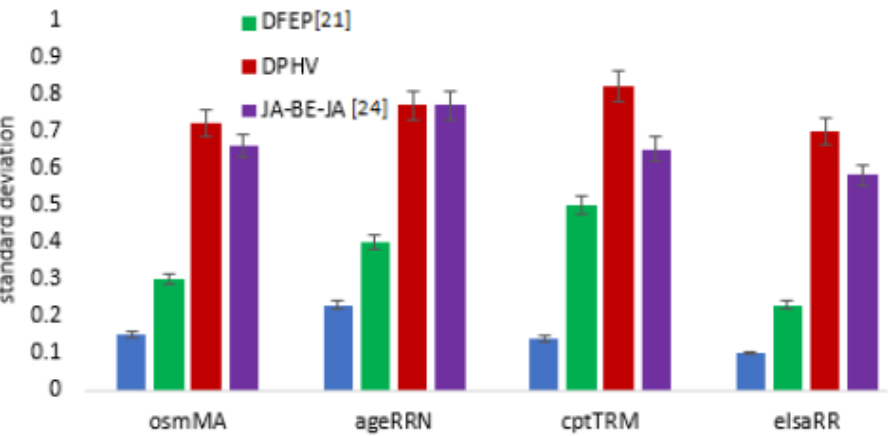

Figure 16

Comparison between DPHV, DFEP [21], JA-BE-JA [24] and Greedy [23] $(k=100)$ 